\title{
TP53 gain-of-function mutation promotes inflammation in glioblastoma
}

\author{
Seok Won Ham ${ }^{1} \cdot$ Hee-Young Jeon ${ }^{1,2} \cdot$ Xiong Jin $^{1,2}$ • Eun-Jung Kim ${ }^{1,2} \cdot$ Jun-Kyum Kim ${ }^{1,2}$ - Yong Jae Shin ${ }^{3,4} \cdot$ Yeri Lee $^{3}$.

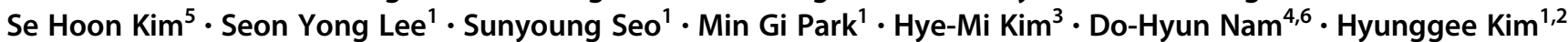

Received: 1 August 2017 / Revised: 9 April 2018 / Accepted: 2 May 2018 / Published online: 21 May 2018

(c) ADMC Associazione Differenziamento e Morte Cellulare 2018

\begin{abstract}
Glioblastoma (GBM), the most severe and common brain tumor in adults, is characterized by multiple somatic mutations and aberrant activation of inflammatory responses. Immune cell infiltration and subsequent inflammation cause tumor growth and resistance to therapy. Somatic loss-of-function mutations in the gene encoding tumor suppressor protein p53 (TP53) are frequently observed in various cancers. However, numerous studies suggest that TP53 regulates malignant phenotypes by gain-of-function (GOF) mutations. Here we demonstrate that a TP53 GOF mutation promotes inflammation in GBM. Ectopic expression of a TP53 GOF mutant induced transcriptomic changes, which resulted in enrichment of gene signatures related to inflammation and chemotaxis. Bioinformatics analyses revealed that a gene signature, upregulated by the TP53 GOF mutation, is associated with progression and shorter overall survival in GBM. We also observed significant correlations between the TP53 GOF mutation signature and inflammation in the clinical database of GBM and other cancers. The TP53 GOF mutant showed upregulated $\mathrm{C}-\mathrm{C}$ motif chemokine ligand 2 (CCL2) and tumor necrosis factor alpha (TNFA) expression via nuclear factor kappa $\mathrm{B}(\mathrm{NFKB})$ signaling, consequently increasing microglia and monocyte-derived immune cell infiltration. Additionally, TP53 GOF mutation and CCL2 and TNFA expression correlated positively with tumorassociated immunity in patients with GBM. Taken together, our findings suggest that the TP53 GOF mutation plays a crucial role in inflammatory responses, thereby deteriorating prognostic outcomes in patients with GBM.
\end{abstract}

\section{Introduction}

Glioblastoma (GBM), the most fatal brain cancer, accounts for $82 \%$ patients with malignant glioma [1]. Radiotherapy and chemotherapy after surgical resection are used as

Edited by P. Salomoni

Electronic supplementary material The online version of this article (https://doi.org/10.1038/s41418-018-0126-3) contains supplementary material, which is available to authorized users.

$\triangle$ Do-Hyun Nam

nsnam@skku.edu

$\triangle$ Hyunggee Kim

hg-kim@korea.ac.kr

1 Department of Biotechnology, College of Life Sciences and Biotechnology, Korea University, Seoul 02841, Republic of Korea

2 Institute of Animal Molecular Biotechnology, Korea University, Seoul 02841, Republic of Korea standard therapy; however, owing to the recurrence of GBM, the 5-year survival rate is less than 5\% [1,2].

Genome-wide sequencing has revealed core mutations in GBM, including amplification in epidermal growth factor receptor $(E G F R)$, and mutations in phosphatase and tensin homolog and TP53 [3]. Transcriptomic analysis demonstrated that patients with GBM exhibit different gene expression profiles, and that GBM can be classified into several subtypes [4]. Nevertheless, the development of individual mutation-specific therapeutic regimens remains poorly investigated. Presently, radiotherapy, combined with

Research Institute for Future Medicine, Samsung Medical Center, Seoul 06351, Republic of Korea

4 Department of Neurosurgery, Samsung Medical Center, Seoul 06351, Republic of Korea

5 Department of Pathology, College of Medicine, Yonsei University, Seoul 03722, Republic of Korea

6 Department of Health Sciences and Technology (SAIHST), Sungkyunkwan University School of Medicine, Seoul 06351, Republic of Korea 
temozolomide or bevacizumab therapy, prolongs patient survival by an average of 2 months [5]. Therefore, understanding the relationship between somatic mutations and tumor characteristics is important for custom-designing individualized therapy for patients with GBM.

The tumor suppressor TP53 is a regulator of cell cycle, apoptosis, and senescence. Various stresses, such as replicative or oxidative stress, and anti-tumor therapy, activate TP53 (hereafter termed p53) by increasing its stability [6]. The activated p53 acts as a transcriptional regulator of its downstream genes such as regulators of cell cycle

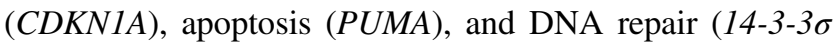
and XPC). TP53 is involved in the regulation of autophagy and metabolism, indicating that $\mathrm{p} 53$ performs a wide range of functions [7].

Somatic alterations in TP53 are the most extensively investigated genetic variations. TP53 mutations have been detected in $>90 \%$ patients with ovarian serous cystadenocarcinoma and pulmonary squamous cell carcinoma [8, 9]. Approximately $30 \%$ patients with GBM harbor TP53 mutations, whereas mutations are rarely detected in thyroid carcinoma and renal clear cell carcinoma [3, 10, 11]. TP53 mutations are predominantly point mutations, which lead to amino acid substitutions in the DNA binding domain (DBD). In particular, substitution of "hotspot" arginine residues within the DBD such as R175, R248, and R273, which bind directly to DNA, prevents a TP53 loss-of-function (LOF) mutant from functioning as a transcription factor [6].

Recent findings show that mutant p53 gains de novo functions that do not occur in wild-type (WT) p53. Previous studies suggest that the gain-of-function (GOF) mutant TP53 contributes to tumor malignancies by promoting proliferation, tumor forming ability, invasiveness, and angiogenesis [12]. Additionally, inhibition of the p53 GOF mutant suppressed cancer progression, indicating that mutant p53 can act as an independent oncoprotein [13, 14]. However, several studies on GOF mutations have compared phenotypes of cell lines with different TP53 status; therefore, it is plausible that differences in these phenotypes are caused not only by the p53 GOF mutation per se, but also by the different genetic background of each cell line. Additionally, several genes, which specifically bind to mutant p53, or genes that can be regulated by TP53 GOF mutations, are known; however, a comprehensive understanding of how cell physiology is altered by TP53 GOF mutations is still limited [12]. A bioinformatics-based unbiased approach can clarify the relationship between the transcriptomic changes caused by TP53 GOF mutations and the resulting phenotypic differences; such an approach can also demonstrate clinical relevance using the patient gene expression database.

Inflammation regulates cancer progression in early and late stages [15], and inflammatory conditions increase the risk of cancer at the onset of the tumor. The most well-studied model is inflammatory bowel disease, which promotes tumorigenesis in colorectal carcinoma by causing DNA damage and providing growth-promoting cytokines [16]. In the late stage, activation of nuclear factor kappa B (NFKB) promotes cell proliferation and resistance to chemotherapy [17]. NFkB releases cytokines such as tumor necrosis factor alpha (TNF $\alpha$ ), interleukins (ILs), and chemokines.

Cytokines downstream of $\mathrm{NFKB}$ promote chemotaxis and convert myeloid cells of the M1-like tumor-suppressing phenotype to the M2-like tumor-promoting phenotype [15]. Myeloid cells with M2-like phenotypes that are associated with tumor tissues, such as tumor-associated macrophages (TAMs) and tumor-associated neutrophils (TANs), promote angiogenesis, proliferation, survival, and invasion via secretion of cytokines [18, 19]. Blocking of TAM-like polarization by inhibiting colony stimulating factor-1 receptor suppresses cancer progression, implying that TAMs play an essential role in tumor maintenance [20,21].

Here we demonstrate that the TP53 GOF mutation promotes inflammation, which has a profound effect on patient prognosis. The genetic signature, upregulated by the TP53 GOF mutant, was closely related to the grade of the brain tumors and displayed positive correlations with inflammation-related gene signatures in the patient gene expression dataset. In particular, we observed that the TP53 GOF mutant promotes microglia and myeloid-derived immune cell infiltration by upregulating CCL2 and TNFA expression, and that the TP53 GOF mutation is associated with the tumor-associated myeloid signature. Our data also show that the association between TP53 mutation and inflammatory response is universal across cancer types, indicating that $T P 53 \mathrm{GOF}$ mutation may significantly affect cancer progression.

\section{Results}

\section{TP53 status correlates with malignant histology in GBM xenograft tumor}

To investigate the genetic factors responsible for the histological features associated with GBM malignancies, we transplanted patient-derived GBM cells in Balb/c nu/nu mice. Compared to $19 \mathrm{NS}$ and $84 \mathrm{NS}$ tumors, $157 \mathrm{NS}$, 528NS, and MD13 tumors showed an increase in tumor initiating capacity, size, cellular density, necrosis, and hemorrhage (Fig. 1a, b, Supplementary Fig. S1a). Somatic mutation in TP53 is the most prominent genetic abnormality that is closely related to malignancy; TP53-mutant tumors exhibit higher accumulation of p53 than do tumors with $T P 53^{W T}$ [12] cDNA sequencing showed that all five GBM cell lines harbored a substitution in the P72R amino acid, 


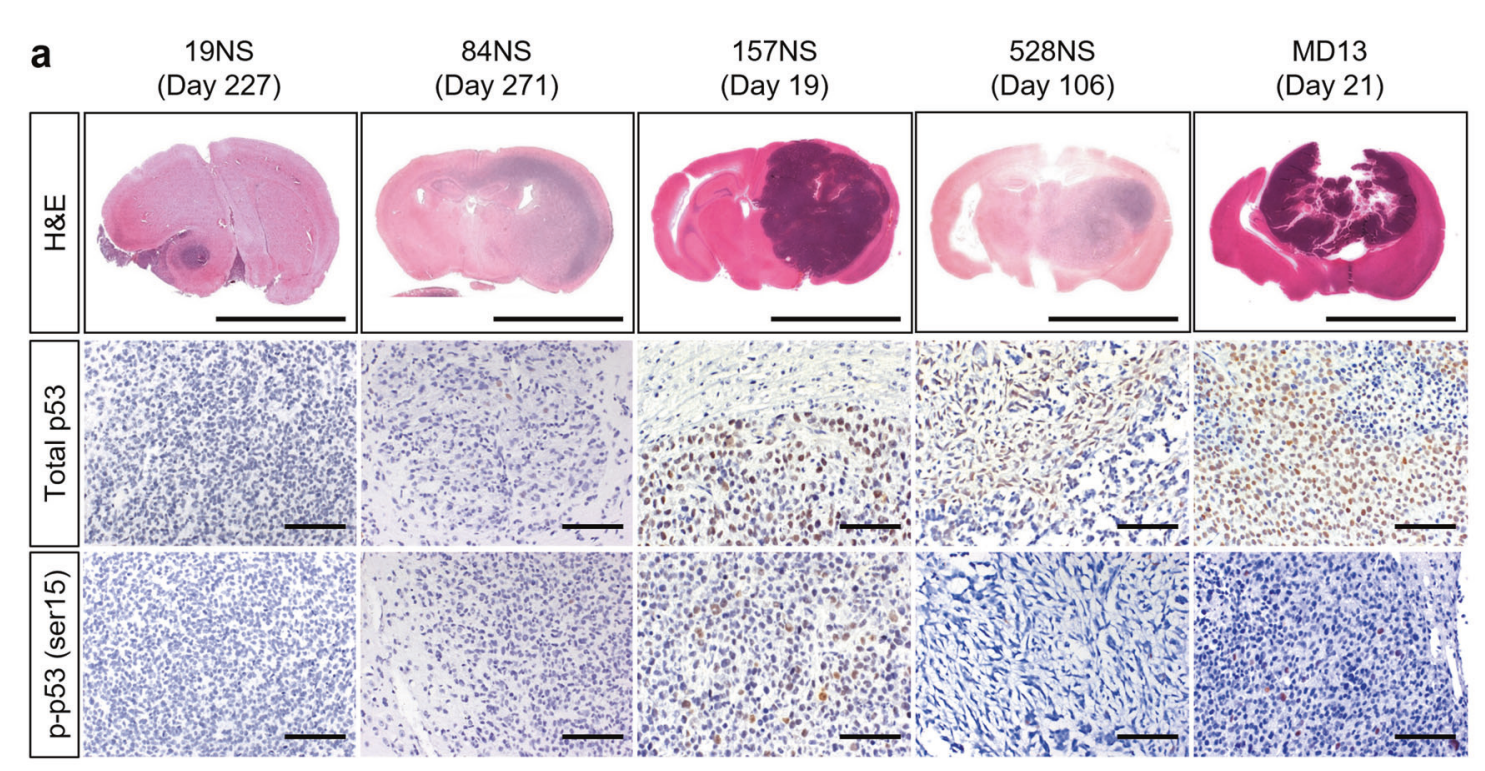

b Day 150 post engraftment $\left(1.0 \times 10^{5}\right.$ cells per mouse $)$

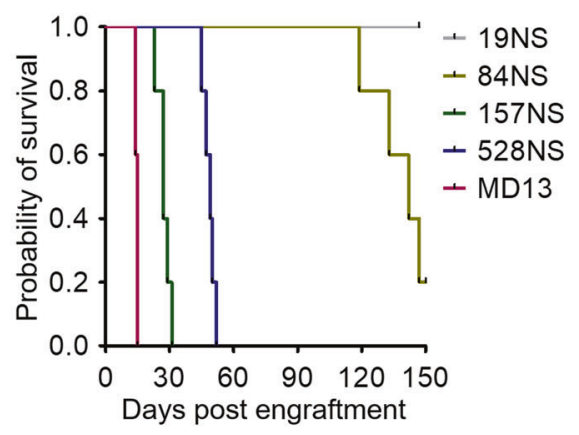

\begin{tabular}{cccc}
\hline Cell line & $\begin{array}{c}\text { Tumor } \\
\text { formation }\end{array}$ & $\begin{array}{c}\text { Mean } \\
\text { survival }\end{array}$ & SEM \\
\hline 19NS & $0 / 4$ & - & - \\
84NS & $4 / 5$ & 138.2 & 5.60 \\
157NS & $5 / 5$ & 27.4 & 1.33 \\
528NS & $5 / 5$ & 48.6 & 1.21 \\
MD13 & $5 / 5$ & 14.6 & 0.24 \\
\hline
\end{tabular}
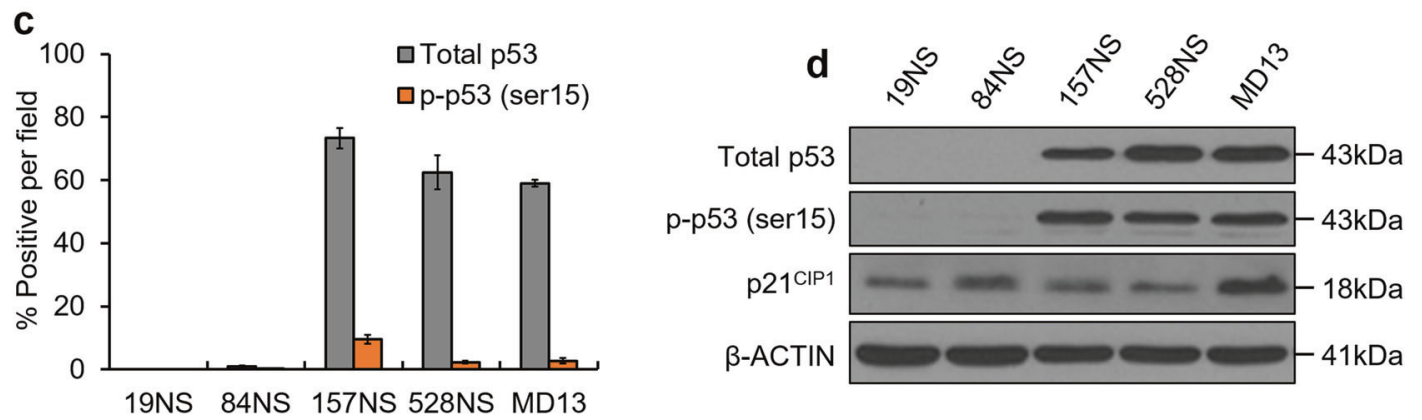

Fig. 1 The distinct histological features of patient-derived GBM are associated with p53 expression. a Representative images of H\&E staining and IHC. The date displayed above each photo indicates the time of mouse sacrifice. $\mathbf{b}$ Tumor forming capacities of patient-derived GBM. A Kaplan-Meier survival plot of mice grafted with the same number of each GBM cell lines (left), and a table displaying the mean

which is one of the common single-nucleotide polymorphisms with intact function; therefore, this mutation is hereafter considered as WT (Supplementary Fig. S1b-d) [22]. Unlike 19NS and 84NS, 157NS, 528NS, and MD13 harbor additional amino acid substitutions, particularly the R248L substitution in 157NS and MD13, this is one of the common hotspot DBD mutations (Supplementary overall survival (right). c Quantification of IHC provided in a. d Western blot analysis for total p53, phosphorylated p53 (p-p53, ser15), and $\mathrm{p} 21^{\mathrm{CIP} 1}$. Representative microscopic images of $\mathrm{H} \& \mathrm{E}$ and IHC are magnified $6 \times($ scale bar $=5 \mathrm{~mm})$ and $200 \times($ scale bar $=100 \mu \mathrm{m})$, respectively. The bar graph represents the mean \pm SEM

Fig. S1b-d) [6]. Immunohistochemistry (IHC) revealed that the 157NS, 528NS, and MD13 tumors show relatively high p53 accumulation, whereas the 19NS and 84NS tumors showed negligible p53 expression (Fig. 1a, c). Few cells of the 157NS, 528NS, and MD13 tumors were positive for serine 15-phosphorylated p53 (p-p53), a damage-induced active form of p53. Western blot analysis showed high 

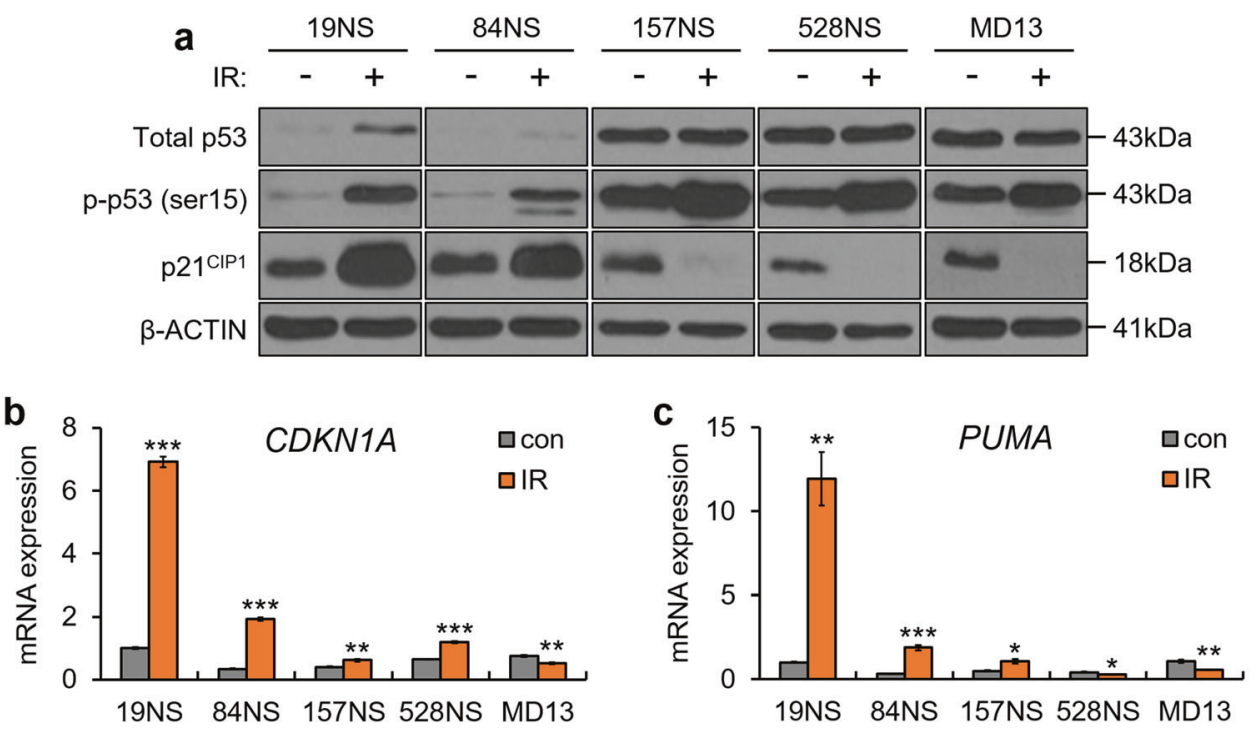

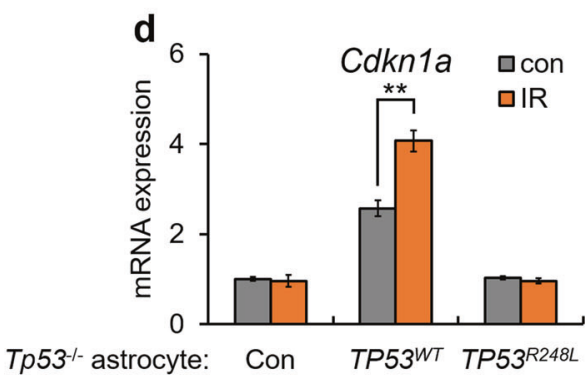

Fig. 2 Targeted sequence analysis shows TP53 LOF mutations in patient-derived GBM cell lines. a Western blot analysis for total p53, p-p53 (ser15), and p21 ${ }^{\mathrm{CIP} 1}$ after treatment with $10 \mathrm{~Gy}$ IR. b qPCR analysis, showing mRNA expression of CDKN1A and PUMA after treatment with 10 Gy IR. $\mathbf{c}$ qPCR analysis, showing $C D K N 1 A$ mRNA level after treatment with 10 Gy IR. d qPCR analysis, showing mRNA

levels of total and phosphorylated p53 in 157NS, 528NS, and MD13, but not those of $\mathrm{p} 21^{\mathrm{CIP} 1}$, a prominent downstream target of p53 (Fig. 1d). These results indicate that tumors harboring a TP53 hotspot mutation display malignant histological features and accumulate p53.

\section{GBM xenograft tumors possess TP53 LOF somatic mutation}

We investigated the activity of p53 as a transcriptional regulator to determine whether the $T P 53^{R 248 L}$ is a LOF mutant. $\mathrm{p} 53$ phosphorylation and $\mathrm{p} 21^{\mathrm{CIP} 1}$ levels increased in 19NS and 84NS after ionizing radiation (IR)-mediated DNA damage, but not in 157NS, 528NS, and MD13, which showed low levels of $\mathrm{p} 21^{\mathrm{CIP} 1}$, similar to the results of previous studies (Fig. 2a) [7, 23-25]. The mRNA levels of CDKNIA and PUMA increased more than two-fold in IRexposed 19NS and 84NS; however, these levels changed slightly in $157 \mathrm{NS}, 528 \mathrm{NS}$, and MD13 (Fig. 2b, c). Furthermore, when ectopically expressed in astrocytes derived

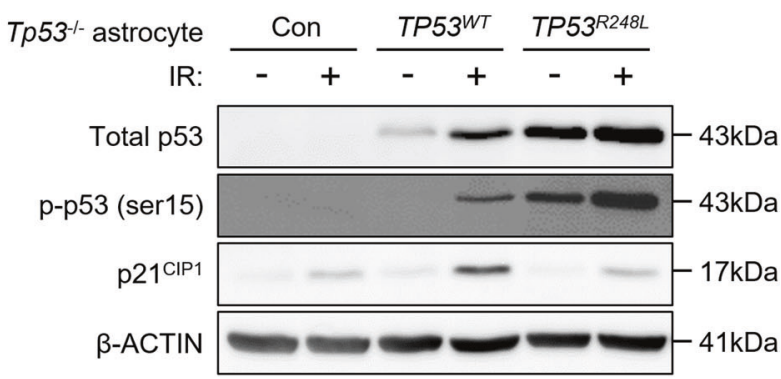

expression of Cdknla after treatment with 10 Gy IR, in $T p 53^{-1-}$ astrocytes transduced with $T P 53^{W T}$ or $T P 53^{R 248 L}$. e Western blot analysis for total p53, p-p53 (ser15), and p2 ${ }^{\mathrm{CIP} 1}$ after treatment with 10 Gy IR, in $T p 53^{-/-}$astrocytes transduced with $T P 53^{W T}$ or $T P 53^{R 248 L}$. The bar graph data represent the mean \pm SEM $(* P<0.05 ; * * P<0.01$; $* * * P<0.001, n=3$ )

from $T P 53^{-/}$mouse, TP5 $3^{R 248 L}$ did not promote $\mathrm{p} 21^{\mathrm{CIP} 1}$ expression during IR (Fig. 2d, e). Our results demonstrate that expression of the TP53 mutation in 157NS and MD13 does not activate the downstream signals similar to the WT, which is a feature of LOF mutants.

\section{$T P 53^{R 248 L}$ upregulates inflammation-related and chemotaxis-related transcriptome}

The $T P 53^{R 248 L}$-expressing xenograft tumors showed features independent of the deficiency of WT p53 activity. Therefore, we assumed that the mutant TP53 affected GBM properties via de novo GOF. To examine the transcriptomic changes mediated by the TP53 GOF, we first used RNA sequencing after overexpressing $T P 53^{R 248 L}$ in $19 \mathrm{NS}$ $\left(T P 53^{W T}\right)$ (Supplementary Fig. S2a). Among the differentially expressed genes, the expression levels of which are increased by $T P 53^{R 248 L}$ overexpression, we selected genes with $>2$-fold increase in expression and a probability $\left(P_{\mathrm{A}}\right)$ of $\geq 0.7$; we considered these genes to be signature of 

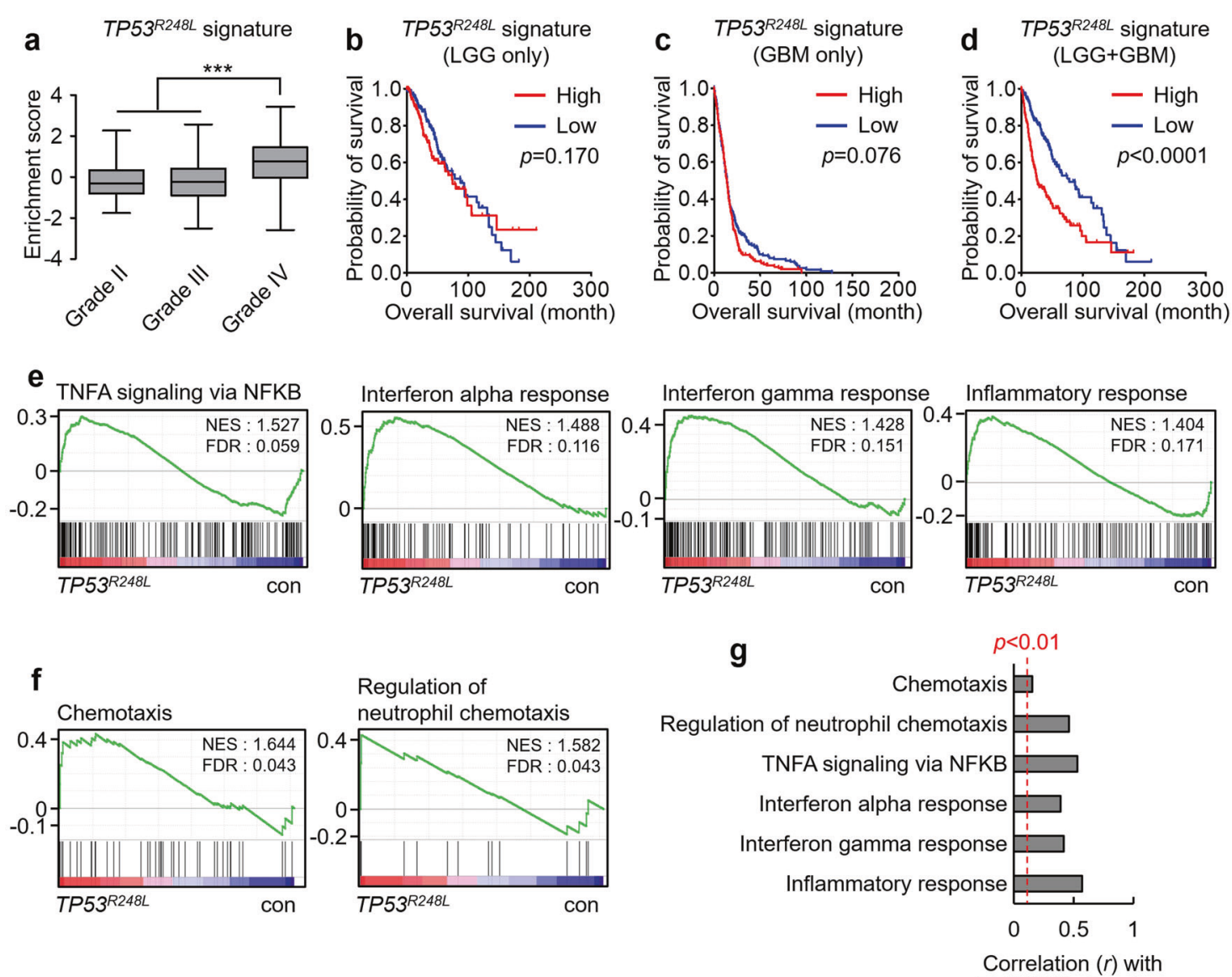

h

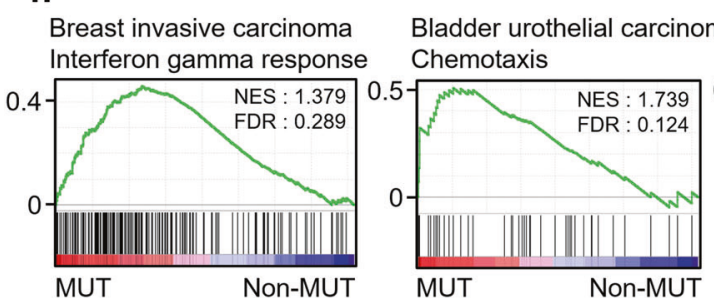

Fig. $3 T P 53^{R 248 L}$ mutation promotes the progression of GBM and enriches inflammation-related signatures. a Tumor grade-dependent enrichment of the $T P 53^{R 248 L}$ signature in patients with low-grade glioma or GBM $(* * * P<0.001$; one-way ANOVA). b-d Kaplan-Meier survival plots showing the overall survival of patients with respect to the $T P 53^{R 248 L}$ signature enrichment. e GSEA demonstrating the enrichment of inflammation-related signatures in $T P 53^{R 248 L}$-overexpressing 19NS GBM cell line. f GSEA showing the

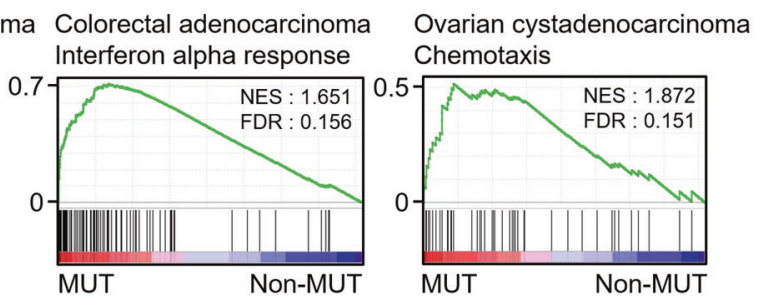

enrichment of chemotaxis-associated signatures in $T P 53^{R 248 L}$-overexpressing 19NS GBM cell line. g Correlations between TP53 $3^{R 248 L}$ signature and inflammation/chemotaxis-related signatures in the TCGA GBM patient expression dataset. h GSEA showing the enrichment of inflammation/chemotaxis-related signatures in various types of cancer with TP53 DNA binding domain (DBD)-mutated (MUT) or non-mutated (non-MUT)

TP53 $3^{R 248 L} \quad$ (Supplementary Fig. S2, Supplementary Table 1). A single-sample gene set enrichment analysis (ssGSEA), performed using a gene expression dataset of low-grade glioma (LGG) or GBM patients at The Cancer Genome Atlas (TCGA), revealed that the $T P 53^{R 248 L}$ signature was relatively enriched in grade IV patients (Fig. 3a). $T P 53^{R 248 L}$ signature enrichment did not affect the overall survival of patients with LGG (Fig. 3b). Among patients with GBM, the group with relatively enriched $T P 53^{R 248 L}$ signature, showed shorter overall survival, although this was

not statistically significant (Fig. 3c). Conversely, the same analysis, conducted on the combined dataset of patients with LGG and GBM, showed that the overall survival rate of the $T P 53^{R 248 L}$ signature-enriched group was considerably reduced (Fig. 3d), indicating that the $T P 53^{R 428 L}$ signature is closely related to tumor aggressiveness.

To better understand the biological significance of the transcriptomic changes that occur during TP53 $3^{R 248 L}$ expression, we performed GSEA using the RNA sequencing results and hallmark signatures. Interestingly, 

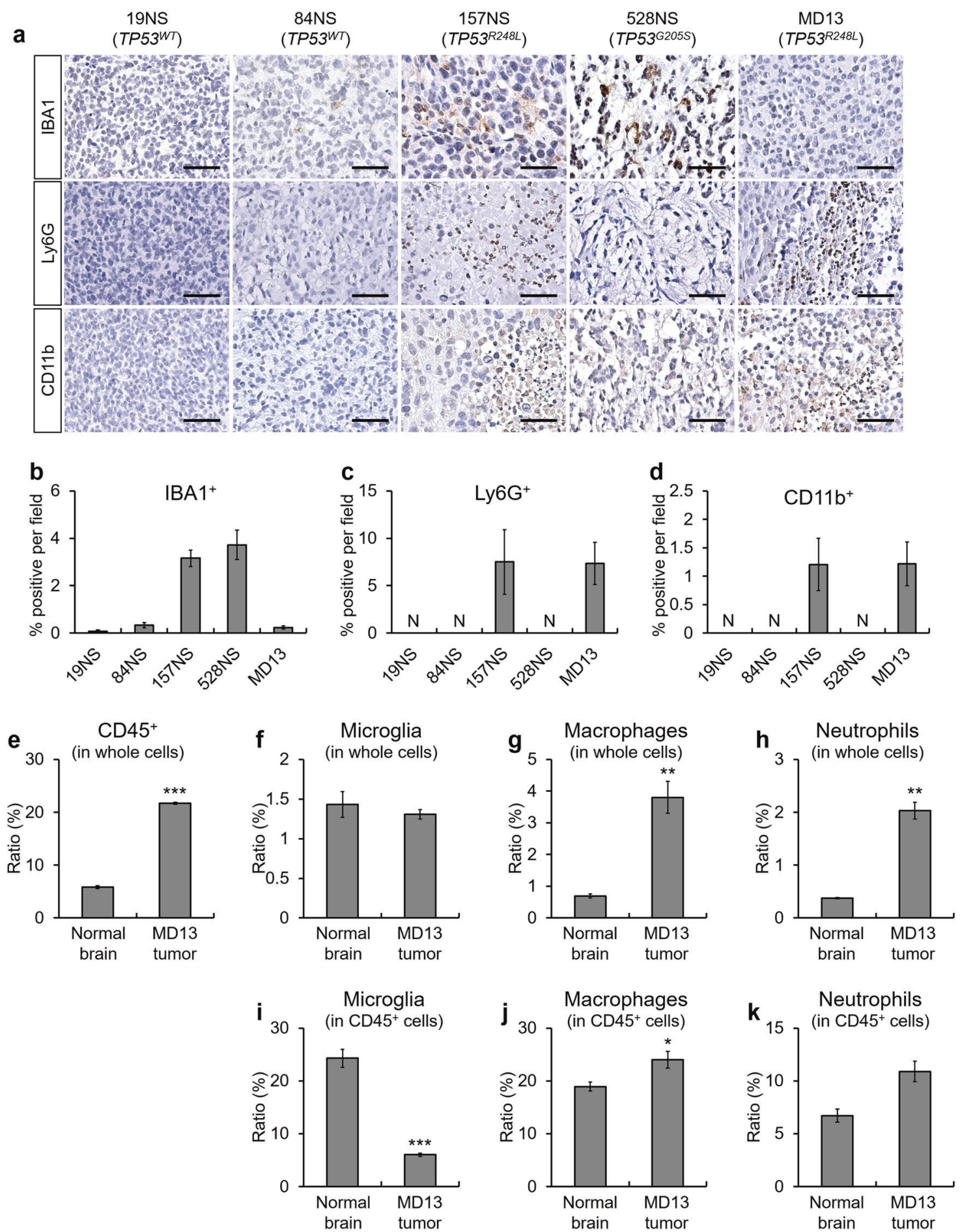

Fig. $4 T P 53^{R 248 L}$ xenograft tumor shows high immune cell population infiltration. a Representative IHC images showing immune cell markers including IBA1, Ly6G, and CD11b. Three mice per cell lines were used except for 19NS. Brain tumor tissue from one mouse was used for 19NS because of its low tumor-forming ability (Fig. 1b). Representative microscope images are magnified $200 \times$ (scale bar $=$

inflammation-related signatures, including TNFA via NFkB signaling, and interferon and inflammation responses, were enriched in $19 \mathrm{NS}$ overexpressing TP53 $3^{R 248 L}$ (Fig. 3e).
$100 \mu \mathrm{m}) . \mathbf{b}-\mathbf{d}$ Quantification of IHC for the composition of IBA1 ${ }^{+}$, Ly $6 \mathrm{G}^{+}$, and $\mathrm{CD} 11 \mathrm{~b}^{+}$immune cells, respectively. e-k Flow cytometry measurement of immune cell composition in the MD13 xenograft brain tumor and normal brain. Dot plots showing the results of the flow cytometry analyses are shown in Supplementary Fig. S5. The bar graphs represent the mean $\pm \mathrm{SEM}$. $\mathrm{N}$ indicates no detection

Additionally, the chemotaxis-related gene signatures were enriched (Fig. 3f), highlighting the relationship between the TP53 GOF mutation and inflammation. 

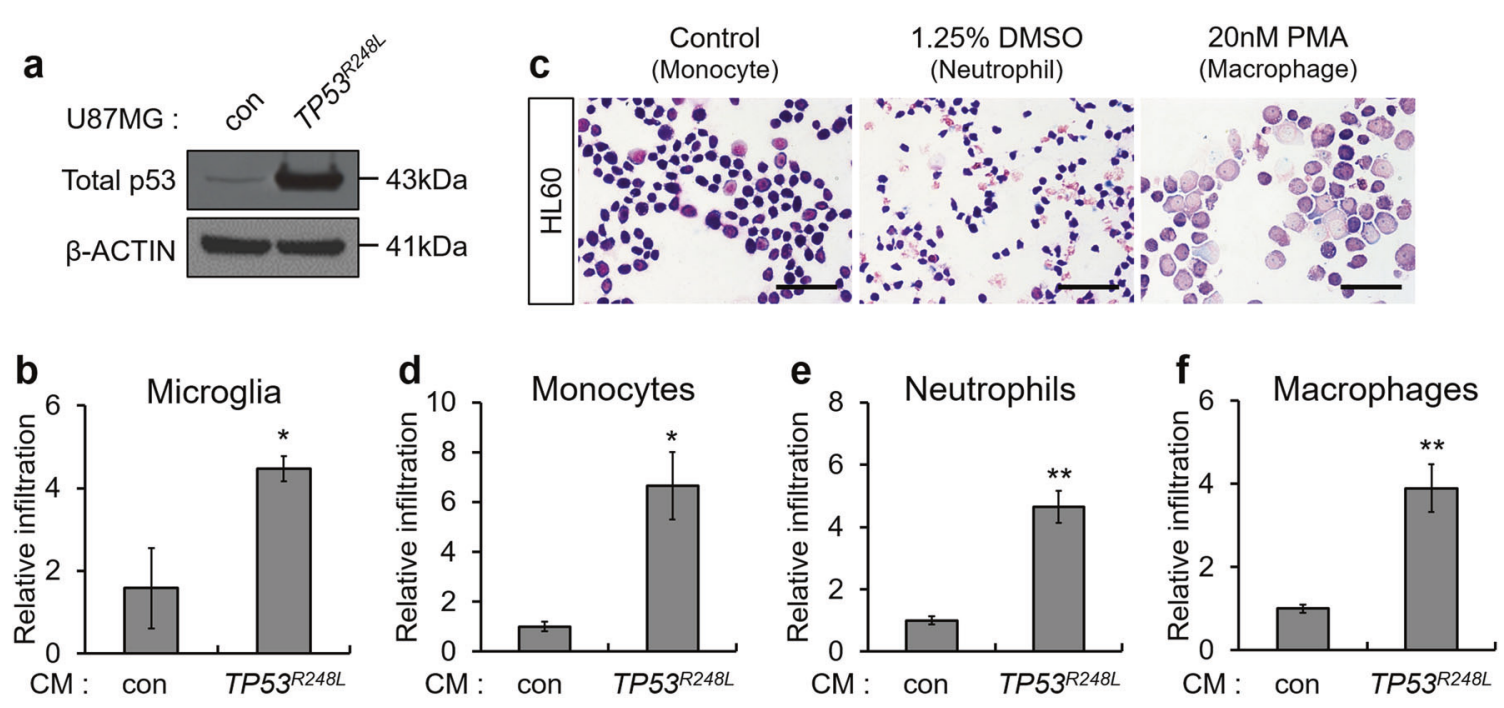

\section{g}
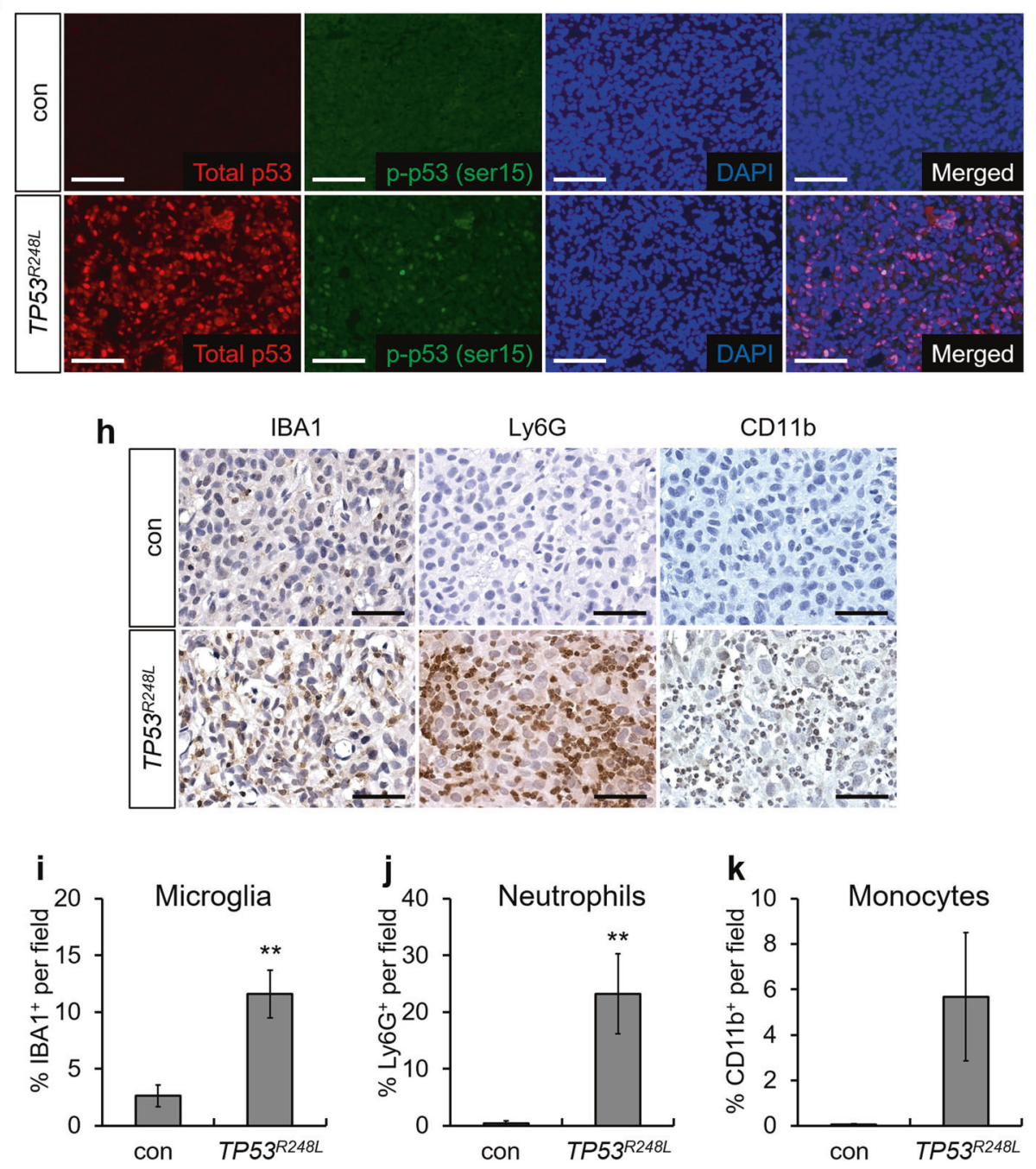

Next, we examined whether the $T P 53^{R 248 L}$-mediated transcriptomic changes, which activate inflammation-related responses, recapitulate the changes in patient expression profile. ssGSEA using the TCGA GBM patient dataset showed that the enrichment scores of inflammation-related and chemotaxis-related gene signatures correlated positively 
Fig. 5 Ectopic expression of $T P 53^{R 248 L}$ promotes immune cell infiltration. a Western blot analysis showing p53 expression in $T P 53^{R 248 L}$ overexpressing U87MG cell line. b Relative infiltration rate of BV2 microglia grown in conditioned medium $(\mathrm{CM})$ generated using control U87MG and TP53 $3^{R 248 L}$-overexpressing U87MG cell lines $(n=3)$. c Differentiation of HL60 to neutrophils and macrophages was confirmed by Diff-Quik staining (magnification $400 \times$, scale bar $=50 \mu \mathrm{m}$ ). d-f Relative infiltration rate of HL60-derived monocytes, neutrophils, and macrophages grown in CM from control U87MG and TP53 $3^{R 248 L_{-}}$ overexpressing U87MG cell lines $(n=3)$. g Representative microscopic images of fluorescent IHC showing total p53 and p-p53 (ser15) in orthotopically transplanted tumors generated using TP53 $3^{R 248 L_{-}}$ overexpressing U87MG cell line (magnification 200x, scale bar $=100$ $\mu \mathrm{m}) . \mathbf{h}$ Representative microscopic images of IHC showing expression of immune cell markers such as IBA1, Ly6G, and CD11b (magnification $200 \times$, scale bar $=100 \mu \mathrm{m}$ ). $\mathbf{i}-\mathbf{k}$ Quantification of IHC showing the composition of IBA $1^{+}, \mathrm{Ly}_{6 \mathrm{G}}{ }^{+}$, and $\mathrm{CD} 11 \mathrm{~b}^{+}$cells. The bar graphs represent mean \pm SEM $(* P<0.05 ; * * P<0.01 ; * * * P<0.001)$

with that of the TP53 $3^{R 248 L}$ signature (Fig. $3 \mathrm{~g}$ ). Next, we accessed the TCGA expression datasets for cancer types with frequent TP53 mutation. GSEA showed that the immune response-related signature was prominently enriched in groups possessing the TP53 DBD mutation (Fig. 3h). Therefore, it is likely that TP53 GOF mutation can cause immune-related pathological differences in GBM and other cancers.

\section{TP53 ${ }^{R 248 L}$ GBM xenograft tumors display higher intratumoral immune cell infiltration}

After identifying the $T P 53^{R 248 L}$-induced transcriptomic changes involved in inflammation and chemotaxis, we investigated whether the TP5 $3^{R 248 L}$-expressing tumors show increased presence of immune cells. We immunohistochemically assessed the markers of four different types of immune cells: IBA1 (microglia), F4/80 (macrophage), Ly6G (neutrophil), and CD11b (monocyte-derived lineage) (Fig. 4a). Tumors with TP53 ${ }^{W T}$ from $19 \mathrm{NS}$ and $84 \mathrm{NS}$ cells showed scant immune cell infiltration, whereas markedly elevated immune cell infiltration was detected in the 157NS, 528NS, and MD13 tumors (Fig. 4a-d, Supplementary Fig. S3). Patient-derived GBM lines, such as MD30, 83NS, and $1123 \mathrm{NS}$, also harbor $T P 53^{R 248 L}$ mutation, with distinct tendencies of hallmark signature enrichment (Supplementary Fig. S4a, b). The tumors from these cell lines also displayed infiltration of various immune cells (Supplementary Fig. S4c-f).

Next, we performed flow cytometry to evaluate tumor immune cell composition and circulation in tumor-grafted mice (Fig. 4e-k, Supplementary Figs. S5-S8). Compared to the normal brain, MD13 xenograft tumors contained large numbers of $\mathrm{CD}^{4} 5^{+}$cells, macrophages (CD45 $\left.{ }^{+} / \mathrm{CD} 11 \mathrm{~b}^{+} / \mathrm{F} 4 / 80^{+}\right)$, and neutrophils $\left(\mathrm{CD} 45^{+} /\right.$

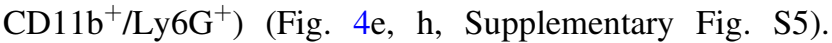

Among $\mathrm{CD} 45^{+}$cells, both microglia and macrophages express IBA1. Hence, we used an antibody against TMEM119 to specifically detect the microglia [26]. The proportion of microglia among the $\mathrm{CD} 45^{+}$cells decreased in the MD13 tumor, whereas those of macrophages and neutrophils increased slightly or did not change significantly, respectively (Fig. 4i, k, Supplementary Fig. S5). In addition, MD13-grafted mice showed increase in the number of circulatory macrophages and neutrophils (Supplementary Fig. S6a-d). Similarly, we observed alterations in immune cell composition in the tumor and circulation of 157NS-grafted mice (Supplementary Figs. S7-S8). These results suggest that $T P 53^{R 248 L}$ actively regulates immune cell recruitment and affects the composition of intratumoral immune cells in GBM, along with systemic inflammation.

\section{$T P 53^{R 248 L}$ promotes chemotaxis}

To investigate whether ectopically expressed $T P 53^{R 248 L}$ promotes chemotaxis, we overexpressed $T P 53^{R 248 L}$ in U87MG because 19NS rarely forms xenograft tumors (Fig. 1b). Transwell migration assay showed an increase in the number of migrating microglia in the $T P 53^{R 248 L}$-conditioned medium $(\mathrm{CM})$ compared to that in $T P 53^{W T}-\mathrm{CM}$ (Fig. 5a, b). To model the infiltration of immune cells of myeloid lineage, we used the HL60 premyelocytic monocyte-like cell line, and differentiated them into neutrophils and macrophages using dimethylsulfoxide (DMSO) and phorbol myristic acid (PMA), respectively (Fig. 5c) [27, 28]. Transwell migration assay showed that $T P 53^{R 248 L}-\mathrm{CM}$ increased migration of HL60-derived immune cells (Fig. 5d-f).

Next, we, investigated whether $T P 53^{R 248 L}$ overexpression stimulated immune cell recruitment in vivo. IHC showed that the infiltration of IBA1 ${ }^{+}$microglia and $\mathrm{Ly}_{6 \mathrm{G}}{ }^{+}$ neutrophils in the $T P 53^{R 248 L}$-overexpressing U87MG tumors was markedly increased compared to that in control tumors, whereas infiltration of $\mathrm{CD}_{11 \mathrm{~b}^{+}}$monocytes did not increase significantly (Fig. 5g-k). Similarly, overexpression of $T P 53^{R 248 L}$ promoted the infiltration of $\mathrm{IBA} 1^{+}$microglia and $\mathrm{F} 4 / 80^{+}$macrophages in $84 \mathrm{NS}$ xenograft tumor (Supplementary Fig. S9). Collectively, our results demonstrate that the GOF mutant TP5 $3^{R 248 L}$ promotes chemotaxis.

\section{TP53 ${ }^{R 248 L}$ upregulates CCL2 and TNFA to promote chemotaxis}

To investigate the mechanism underlying the $T P 53^{R 248 L}$ mediated promotion of chemotaxis, we determined the expression of cytokines with chemotactic ability. Among cytokines, the expression of CCL2 and TNFA markedly increased upon $T P 53^{R 248 L}$ overexpression (Fig. 6a). Considering that $T P 53^{R 248 L}$ promotes infiltration of the mouse- 

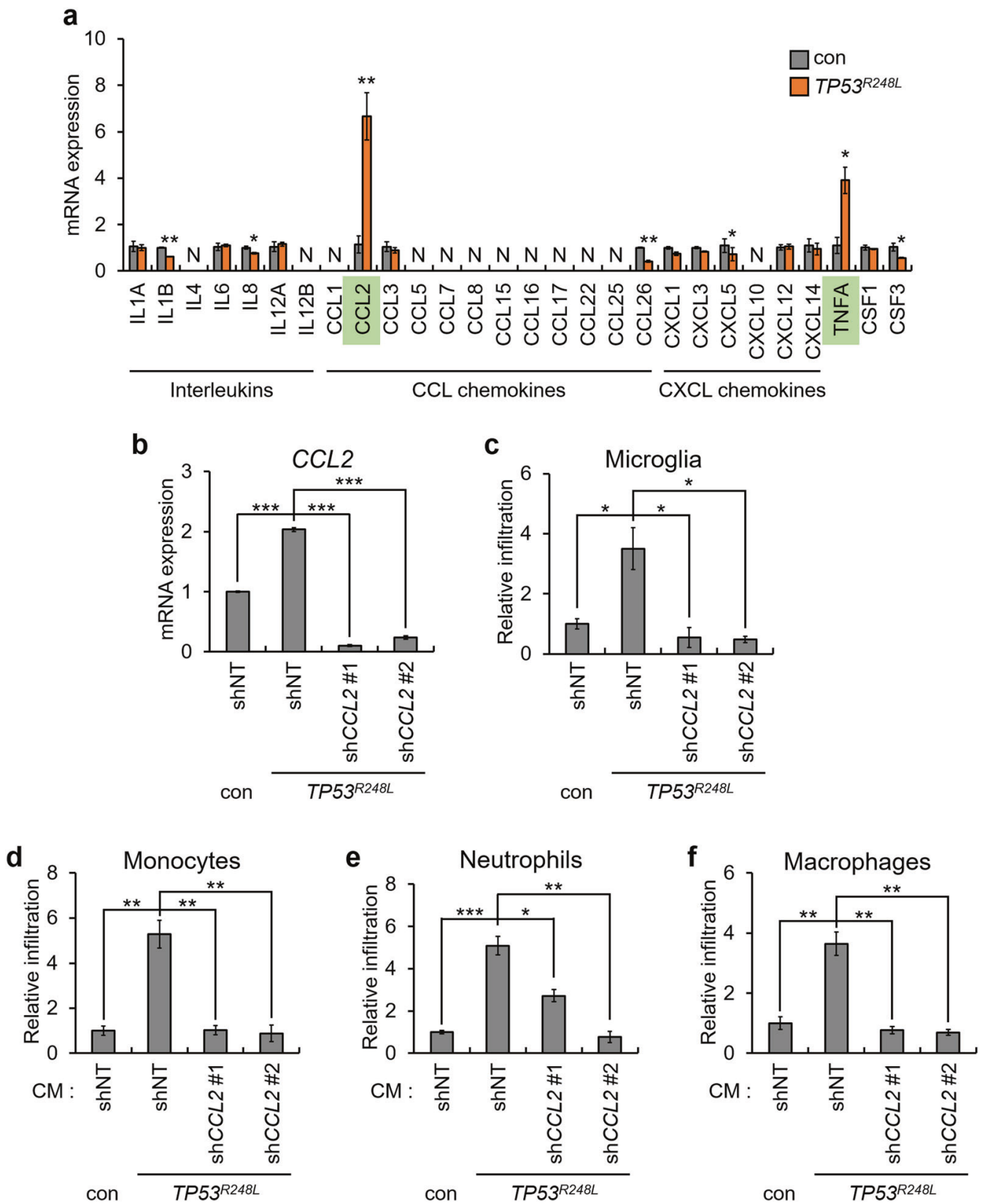

Fig. $6 T P 53^{R 248 L}$ promotes immune cell infiltration by upregulating $C C L 2$ and TNFA. a qPCR analysis showing expression of chemoattractive cytokines in TP53 ${ }^{R 248 L}$-overexpressing U87MG cell line. b qPCR analysis confirming shRNA-mediated knockdown of CCL2 in $T P 53^{R 248 L}$-overexpressing U87MG cell line. shNT indicates nontargeting control shRNA. c-f Relative infiltration rate of BV2

microglia, HL60-derived monocytes, neutrophils, and macrophages grown in CM from a $T P 53^{R 248 L}$-overexpressing U87MG cell line with or without $C C L 2$ knockdown, and control counterpart cells. The bar graphs represent mean \pm SEM $(* P<0.05 ; * * P<0.01$; $* * * P<0.001 ; n=3)$

derived BV2 microglia, and that TNF $\alpha$ lacks interspecies reactivity between human and mouse, we hypothesized that CCL2 predominantly affects chemotaxis [29]. Hence, we knocked down CCL2 using short hairpin RNAs (shRNAs) in $T P 53^{R 248 L}$-overexpressing U87MG cells (Fig. 6b). $T P 53^{R 248 L}$ overexpression-mediated increase in immune cell infiltration was reduced by $C C L 2$ knockdown, indicating that $\mathrm{p} 53^{\mathrm{R} 248 \mathrm{~L}}$ promotes chemotaxis via CCL2 (Fig. $6 \mathrm{c}-\mathrm{f}$ ).

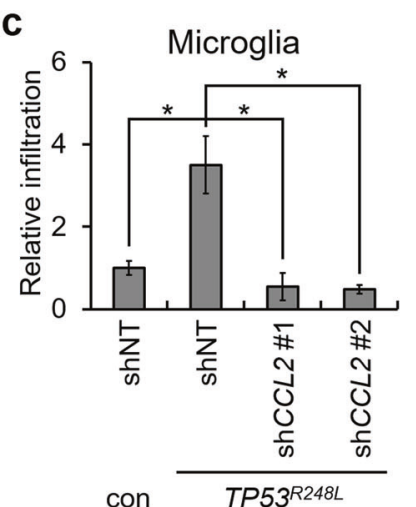

Next, we investigated whether $C C L 2$ knockdown inhibited the progression of $T P 53^{R 248 L}$-expressing tumors. Decrease in the CCL2 expression did not affect proliferation of $T P 53^{R 248 L}$-expressing U87MG cells (Supplementary Fig. S10a). In addition, CCL2 knockdown prolonged mean overall survival of mice grafted with $T P 53^{R 248 L}$-expressing U87MG, although it was not statistically significant (Supplementary Fig. S10b). Thus, our observations suggest that 

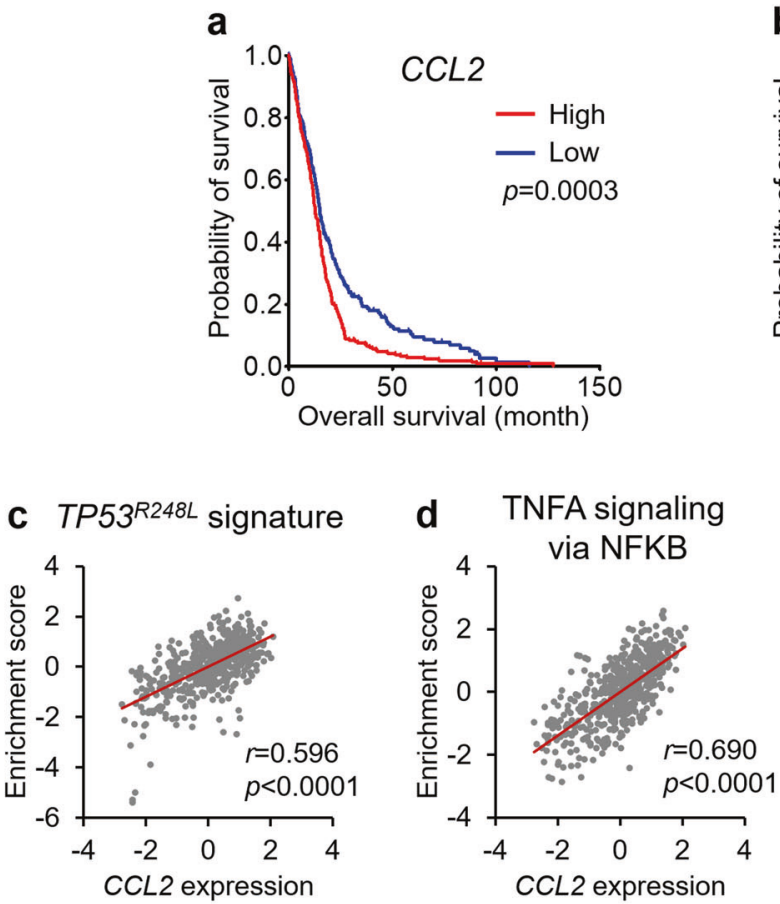

f TAN-related genes

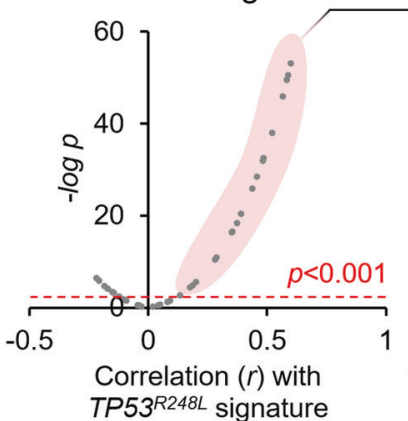

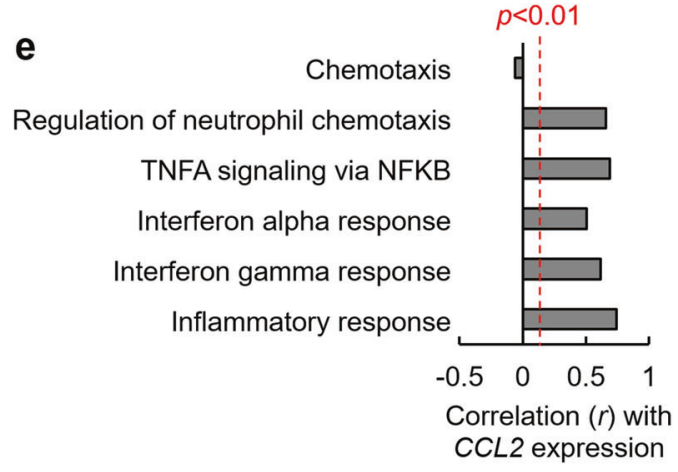

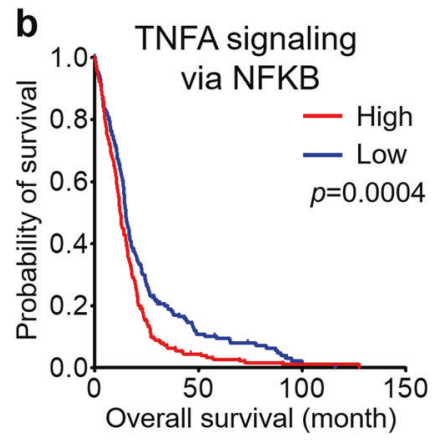

g TAM-related genes

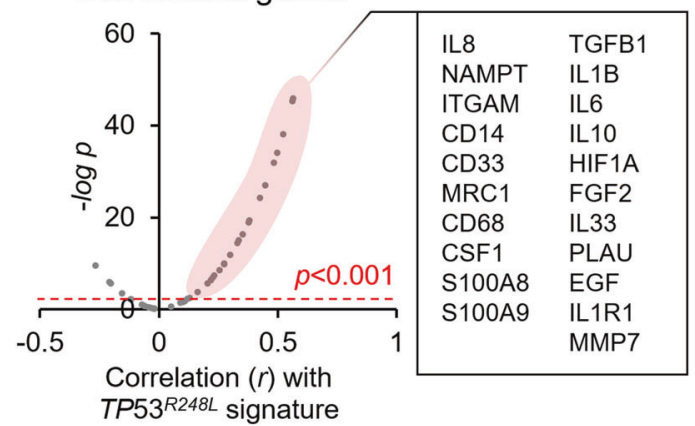

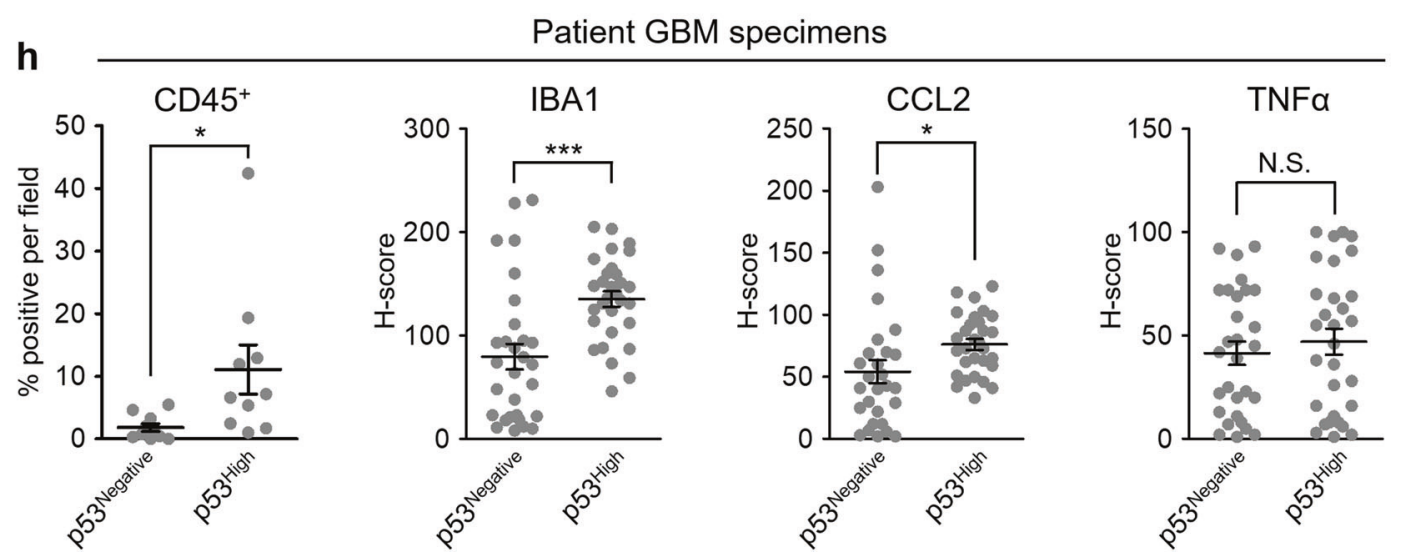

Fig. $7 C C L 2$ and TNFA, induced by $T P 53^{R 248 L}$, affect patient prognosis and tumor-associated myeloid signatures. a, b Kaplan-Meier survival plots showing overall survival of patients with respect to CCL2 expression and enrichment of TNFA signaling via NFKB signature. c, d Correlation between CCL2 expression and enrichment scores of the $T P 53^{R 248 L}$ signature, and TNFA signaling via NFKB signature. e Correlations between $T P 53^{R 248 L}$ signature and inflammation/chemotaxis-related signatures. f, $\mathbf{g}$ Correlations between the TP53 $3^{R 248 L}$ signature and expression of TAN-related and TAMrelated genes. All bioinformatics analyses were performed with the TCGA GBM patient gene set. h Quantification of IHC for CD45, IBA1, CCL2, and TNF $\alpha$ in the patient-derived GBM tissues, grouped by p53 levels $(* P<0.05 ; * * * P<0.001 ; n=10$ for each group) 
$T P 53^{R 248 L}$-mediated increase in CCL2 expression may promote immune cell recruitment, although it is not exclusively responsible for tumor progression.

We investigated the mechanism via which mutant p53 regulates CCL2 expression. Previous studies suggest that p53 ${ }^{\mathrm{WT}}$ suppresses CCL2 expression, while concurrently, mutant p53 may functionally block $\mathrm{p} 53^{\mathrm{WT}}$ in a dominantnegative manner $[6,30]$. Thus, we confirmed that $T P 53^{R 248 L}$ acts as a dominant-negative mutant and suppresses the p53 ${ }^{\mathrm{WT}}$-mediated negative regulation of CCL2 expression. CCL2 mRNA level was not reduced in TP53 ${ }^{W T}$ cells by IRmediated activation of $\mathrm{p} 53$, and the dominant-negative deletion mutant TP53 $\left(T P 53^{D D}\right)$ reduced CCL2 and TNFA expression (Supplementary Fig. S11a-d). These results indicate that CCL2 and TNFA expression levels are not increased by the dominant-negative effect of $T P 53^{R 248 L}$ on $T P 53^{W T}$.

As observed in the transcriptomic analyses, overexpression of $T P 53^{R 248 L}$ induced enrichment of gene signatures related to $\mathrm{NF \kappa B}$ signaling (Fig. 3e). Similarly, a previous study has shown that mutant p53 activates the $\mathrm{NF \kappa B}$ signaling pathway in an inflammatory microenvironment [31]. Thus, we hypothesized that $T P 53^{R 248 L}$ increases CCL2 and TNFA expression via the NFKB signaling pathway. We observed that treatment with the NFKB inhibitor, Bay11-7085, inhibited the $T P 53^{R 248 L}$-mediated increase in CCL2 and TNFA expression, indicating that $T P 53^{R 248 L}$ upregulates CCL2 and TNFA by increasing NFкB signaling (Supplementary Fig. S12a, b).

\section{$T P 53^{R 248 L}$-derived inflammatory responses critically affect patient prognosis}

Since the TP53 ${ }^{R 248 L}$ signature affects patient survival and promotes inflammation, we analyzed the TCGA dataset of patients with GBM to determine the clinical relevance of CCL2 and TNFA expression. CCL2 expression and enrichment of TNFA signaling via NFKB negatively affect patient survival (Fig. 7a, b). CCL2 expression correlated positively with enrichment scores of the $T P 53^{R 248 L}$ and TNFA signaling signature; there was also a significant correlation with signatures associated with inflammation and chemotaxis (Fig. 7c-e).

Next, we determined whether $T P 53^{R 248 L}$ regulated the TAM-like conversion of immune cells to establish a tumorfavoring inflammatory microenvironment [15]. Notably, the expression of TAN-related and TAM-related genes showed a strong positive correlation with the $T P 53^{R 248 L}$ signature enrichment score and CCL2 and TNFA expression in the TCGA dataset of patients with GBM (Fig. 7f, g, Supplementary Fig. S13a-d) [32, 33].

To address the clinical significance of inflammatory responses caused by the TP53 GOF mutations, we performed IHC against CD45, IBA1, CCL2, and TNF $\alpha$ using patient GBM tissues, which are negative ( $\left.\mathrm{p} 53^{\text {Negative }}\right)$ or highly positive $\left(\mathrm{p} 53^{\text {High }}\right)$ for $\mathrm{p} 53$. $\mathrm{p} 53^{\text {High }} \mathrm{GBM}$ tissues exhibited increase in the number of $\mathrm{CD} 45^{+}$cells and IBA $1^{+}$ microglia (Fig. 7h, Supplementary Fig. S14). Similarly, CCL2 expression was higher in the $\mathrm{p} 53^{\text {High }} \mathrm{GBM}$, whereas TNFA expression did not show any difference (Fig. 7h, Supplementary Fig. S14). Furthermore, ssGSEA using the IVY Glioblastoma Atlas Project (IVY GAP) database revealed that the $T P 53^{R 248 L}$ signature correlates positively with inflammation-related signatures in perinecrotic zones and cellular tumor regions (Supplementary Fig. S15). Taken together, our results suggest that the TP53 GOF mutation facilitates inflammatory responses, which may eventually affect tumor progression.

\section{Discussion}

The TP53 LOF mutation results in uncontrolled proliferation and desensitization of cancer cells to therapies; however, the effect of a TP53 mutation on prognostic outcomes is still uncertain for GBM [7]. A clinical study indicated that patients with TP53 mutation are less sensitive to treatment; however, other studies did not indicate any significant reduction in the overall survival rate of patients with TP53 mutations [34-36]. No significant differences were observed in the resistance of several GBM cell lines with TP53 mutation to temozolomide in vitro [37]. Interestingly, TP53 mutations did not affect prognosis; however, the overall survival rate of a group of patients, with overexpression of mutant TP53, was significantly reduced [36]. Similarly, several studies have also shown that overall survival is reduced in patients with high TP53 immunoreactive glioma [38, 39], suggesting clinical significance of the TP53 GOF mutation.

Inflammation accelerates cancer progression in GBM and enables resistance to treatment. Analysis of tissue samples from patients, used for selecting prognostic gene signatures, indicated that predictive gene signatures were mostly associated with inflammatory response [40]. Similarly, increase in the number of neutrophils and inflammatory serum protein levels is associated with poor prognosis [41, 42]. A recent transcriptome-wide analysis revealed that recurrent tumors undergo microenvironmental evolution, which involves increased infiltration of tumor-associated microglia and macrophages, and that the composition of TAM is associated with earlier recurrence [43]. These results indicate that inflammation is important for predicting the severity and treatment outcome of GBM.

Our observations support previous experimental evidence indicating that inflammation and TP53 GOF mutations are important determinants of prognosis in GBM. Overexpression of $T P 53^{R 248 L}$ induced transcriptomic 
alterations, resulting in enrichment of inflammation-related gene signatures. The $T P 53^{R 248 L}$ signature was related to the progression and prognosis of brain tumors. TP53 $3^{R 248 L}$ promoted infiltration of immune cells by CCL2 upregulation via NFKB signaling. In addition, we observed that TP53 GOF mutation increased immune cell infiltration and CCL2 expression in specimens of patients with GBM.

TNF $\alpha$ facilitates tumor vascularization and hemorrhagic necrosis, and also affects therapeutic sensitivity, primarily through NFkB signaling [44]. Recent studies showed that the mesenchymal subtype of GBM is more invasive than the proneural subtype, and is associated with poor prognosis after treatment $[3,45]$. NFKB is a regulator of proneural-mesenchymal transition, maintenance, and resistance to radiation therapy in the mesenchymal subtype of GBM [46, 47]. 157NS and MD13 harbor EGFR mutation (EGFRvIII), which may affect NFKB signaling activity [48]. However, 84NS, which shows less malignant phenotype than 157NS and MD13, also expresses EGFRvIII. In addition, $T P 53^{R 248 L}$ promoted NFKB signaling in $19 \mathrm{NS}$ and U87MG, which do not express EGFRvIII [48]. Thus, our observation that NFKB-related inflammatory response is facilitated by $T P 53^{R 248 L}$ irrespective of EGFR status implies that the TP53 GOF mutation may broadly influence properties of GBM.

We observed that $C C L 2$, upregulated by $T P 53^{R 248 L}$, promotes chemotaxis. CCL2 not only attracts microglia into the GBM, but also promotes the invasion of GBM cells by upregulating IL6 via CCR2 [49]. CCL2 induces the polarization of monocytes to TAM, which was also observed in the patient database analysis [50]. CCL2 recruits regulatory T cells and myeloid-derived suppressor cells to suppress anti-tumor immunity, suggesting that the TP53 GOF mutation may neutralize responses to immunotherapy [51]. Since our experiments were conducted using mice lacking mature lymphoids, the effect of TP53 GOF mutation on tumor-associated acquired immunity should be validated by further studies.

In conclusion, we have shown that the TP53 GOF mutation results in transcriptome-wide changes that promote inflammatory responses and facilitate chemotaxis via NFKBmediated upregulation of CCL2 (Supplementary Fig. S16). Our results indicate that the association between the TP53 GOF mutation and tumor-associated inflammation may be a critically disruptive feature in therapies against GBM.

\section{Materials and methods}

\section{Cell lines and cell culture}

Patient-derived GBM lines, including 19NS, 84NS, 157NS, 528NS, MD13, MD30, 83NS, and 1123NS, were kindly provided by Dr. Ichiro Nakano (Department of Neurosurgery, Ohio State University, USA). These cell lines were cultured in Dulbecco's Modified Eagle's Medium/F12 (Lonza, Basel, SUI) supplemented with $0.2 \%$ B27 (Invitrogen, Carlsbad, CA, USA), $20 \mathrm{ng} / \mathrm{mL}$ epidermal growth factor (R\&D Systems, Minneapolis, MN, USA), $20 \mathrm{ng} / \mathrm{mL}$ basic fibroblast growth factor (bFGF, R\&D Systems), 1\% penicillin/streptomycin (Life Technologies Carlsbad, CA, USA), $2 \mathrm{mmol} / \mathrm{L}$ L-glutamine (Life Technologies), and 50 $\mu \mathrm{g} / \mathrm{mL}$ gentamicin (Mediatech, Manassas, VA, USA). U87MG was purchased from the American Type Culture Collection (Manassas, VA, USA). Tp53 ${ }^{--}$mouse astrocytes were kindly provided by Drs. Ronald DePinho and Lynda Chin (Dana-Farber Cancer Institute, Harvard Medical School, USA) [52]. The BV2 mouse microglial cell line was a gift from Dr. Jong Bae Park (National Cancer Center, Korea). U87MG, $T p 53^{-/-}$astrocytes, and BV2 were cultured in DMEM (Lonza) supplemented with $10 \%$ fetal bovine serum (Biotechnics Research, Lake Forest, CA, USA), 1\% penicillin/streptomycin, $2 \mathrm{mmol} / \mathrm{L}$ L-glutamine, and $50 \mu \mathrm{g} /$ mL gentamicin. HL60 cells, a gift from Dr. Taehoon Chun (Korea University, Korea), were cultured in Roswell Park Memorial Institute-1640 (RPMI-1640; Lonza) supplemented with $5 \%$ fetal bovine serum, $1 \%$ penicillin/streptomycin, 2 $\mathrm{mmol} / \mathrm{L}$ L-glutamine, and $28 \mathrm{mmol} / \mathrm{L}$ HyClone HEPES (General Electronics Healthcare, Little Chalfont, UK).

\section{Cell line construction}

To construct the TP53 $3^{R 248 L}$-overexpressing U87MG and 19NS GBM cell lines and the Tp $53^{-/}$astrocyte cell line, $T P 53^{W T}$ and $T P 53^{R 248 L}$ coding DNA sequences (CDS) were cloned into a pLL CMV puro mammalian lentiviral expression vector. To construct the $T P 53^{R 248 L}$-overexpressed CCL2 knocked down U87MG cell lines, TP53 ${ }^{R 248 L}$ CDS was cloned into a $\mathrm{pLL}$ CMV blast vector. pLKO.1 puro vector-cloned shRNAs targeting CCL2 were purchased from Sigma-Aldrich (St. Louis, MO, USA; shCCL2 \#1: MISSION shRNA NM_002982.3108s21c1; shCCL2 \#2: MISSION shRNA NM_002982.3214s21c1). Non-targeting shRNA (pLKO.1 shNT puro, Sigma-Aldrich) was used as control.

To produce the lentivirus, each expression vector was transfected into transformed human embryonic kidney 293 cells (HEK293T) with second-generation lentiviral packaging plasmids pMD2.G and psPAX2 using the PolyExpress transfection reagent (Excellgen, Rockville, MD, USA). Twenty-four hours after transfection, the culture medium was harvested, incubated with Lenti-X concentrator (Clontech Laboratories, Mountain View, CA, USA), and centrifuged to obtain the concentrated lentivirus. The cells were infected with the lentiviruses in the presence of $6 \mu \mathrm{g} / \mathrm{mL}$ polybrene (Sigma-Aldrich) for $24 \mathrm{~h}$. Overexpression was confirmed by western blot and quantitative polymerase chain reaction (qPCR) (see "qPCR analysis" in Materials and Methods). 


\section{Orthotopic GBM engraftment}

19NS $\left(1 \times 10^{5}\right.$ cells $), 84 \mathrm{NS}\left(1 \times 10^{4}\right.$ cells $), 157 \mathrm{NS}\left(1 \times 10^{3}\right.$ cells), 528NS $\left(1 \times 10^{3}\right.$ cells $)$, MD13 $\left(1 \times 10^{3}\right.$ cells $)$, MD30 $\left(1 \times 10^{3}\right.$ cells $), 83 \mathrm{NS}\left(1 \times 10^{3}\right.$ cells $), 1123 \mathrm{NS}\left(1 \times 10^{3}\right.$ cells $)$, and U87MG $\left(1 \times 10^{5}\right.$ cells) (Fig. 5) and $3 \times 10^{5}$ cells (Supplementary Fig. S10) were harvested, washed, and resuspended with phosphate buffered saline (PBS; SigmaAldrich). Specifically, $1 \times 10^{5}$ cells of $19 \mathrm{NS}, 84 \mathrm{NS}, 157 \mathrm{NS}$, 528NS, and MD13 were prepared for the experiment shown in Fig. 1b. Living cells were counted after staining with trypan blue (Invitrogen), and a defined number of cells was grafted into the caudate putamen (coordinates relative to the bregma; medial-lateral $+2 \mathrm{~mm}$, dorsal-ventral $-3 \mathrm{~mm}$ ) of mice brains using a stereotaxic injection device (JD-SI-02; Jeung Do Bio \& Plant, Seoul, Korea). Tumor-bearing mice were sacrificed when the body weights decreased below 15 g. Cardiac perfusion with PBS, followed by $4 \%$ paraformaldehyde (Sigma-Aldrich) perfusion, was performed to wash out the blood and fix the tissue, respectively. The removed brains were additionally fixed in $4 \%$ paraformaldehyde for $48 \mathrm{~h}$ and paraffin-embedded for further histological analysis. Animal experiments were performed in the specific pathogen-free facility with the approval of the Korea University Institutional Animal Care \& Use Committee (approval no. KUIACUC-2017-35).

\section{Histological analysis of mouse xenograft tumors}

Paraffin-embedded tissues were micro-sectioned at the thickness of $4 \mu \mathrm{m}$. Tissue sections were used for hematoxylin and eosin (H\&E) staining and IHC. H\&E was purchased from Merck, and staining was conducted according to the manufacturer's protocol. Microscopic images were obtained using an Axio Imager M1 upright microscope (Carl Zeiss, Oberkochen, German); the intensity of the IHC stain was quantified using the Metamorph Offline software (Molecular Devices, Sunnyvale, CA, USA).

For chromogenic IHC, tissue sections were boiled for 20 min in sodium citrate buffer ( $\mathrm{pH}$ 6.0) for antigen retrieval; the buffer contained $10 \mathrm{mM}$ sodium citrate (SAMCHUN Chemical, Seoul, Korea) and 0.05\% Tween-20 (LPS Solution, Daejeon, Korea). Endogenous peroxidase was blocked in 9:1 mixture of methanol (DUKSAN Science, Seoul, Korea) and $30 \% \quad \mathrm{H}_{2} \mathrm{O}_{2}$ (Sigma-Aldrich) for $10 \mathrm{~min}$. The tissue sections were sequentially incubated with primary antibodies, washed, incubated with secondary antibodies, and developed using the Vectastain $\mathrm{ABC}$ horse radish peroxidase (HRP) (Vector Laboratories, Burlingame, CA, USA) and DAB peroxidase substrate kits (Vector Laboratories) according to the manufacturer's instructions. The tissue sections were subsequently counterstained using hematoxylin.
For fluorescent IHC, tissues were prepared using the same procedure as that for chromogenic IHC but without blocking the endogenous peroxidase. After incubation with the primary and fluorescence-conjugated secondary antibodies, the tissues were stained with $1 \mu \mathrm{g} / \mathrm{mL} 4^{\prime}, 6$-diamidino-2-phenylindole (Sigma-Aldrich).

\section{IHC of patient GBM specimens}

All GBM specimens were collected with written informed consent under a protocol approved by the Institutional Review Board of the Samsung Medical Center (2010-04001 and 2010-04-004). After obtaining signed informed consent, tumor specimens were obtained from patients with GBM and embedded into a paraffin block.

Tissue sections of paraffin-embedded GBM specimens were stained with antibodies against p53, IBA1, CCL2, and TNF $\alpha$. Vectra 3.0 automated quantitative pathology imaging system (PerkinElmer, Waltham, MA, USA) and inForm ${ }^{\circ}$ Tissue Finder ${ }^{\mathrm{TM}}$ software (PerkinElmer) were used for the quantification. At least three fields were randomly selected for each specimen, while 1-2 fields were analyzed for the small-sized specimen. The $\mathrm{H}$-score was calculated from the percentage of cells $(0-100 \%)$ in each intensity category $(0,1+, 2+$, and $3+)$ after automatic segmentation of cells into cytoplasm and nucleus. The final $\mathrm{H}$-score was on a continuous scale between 0 and $300 . \mathrm{CD}^{4} 5^{+}$cells were quantified by calculating the percentage of IHCpositive cells in the 10 randomly selected fields per specimen.

\section{TP53 protein coding region-targeted sequencing}

RNA samples, extracted from 19NS, 84NS, 157NS, 528NS, and MD13, were processed for cDNA synthesis using the RevertAid first strand cDNA synthesis kit (Thermo Fisher Scientific, Waltham, MA, USA). TP53 coding sequence was amplified from the cDNA samples of each GBM cell line using Ex Taq (Takara, Shiga, Japan) and a set of primers as follows: forward 5'-GTGACACGCTTCCCTGG ATT-3' and reverse 5'-GCTGTCAGTGGGGAACAA GA-3' (45 cycles for $19 \mathrm{NS}$ and $84 \mathrm{NS}$, and 30 cycles for 157NS, 528NS, and MD13). Amplicons from each cDNA sample were cloned into the pGEM T Easy plasmid, followed by bacterial transformation. More than eight colonies harboring the amplicon-cloned plasmid were selected for each GBM line; the plasmid DNA was extracted and sequenced, targeting the cloned TP53 CDS. Nucleotide substitutions were considered mutations only when the substitutions were detected in all the plasmid samples extracted from the colonies of each GBM line. The results, presented as sequencing peaks, were generated using Chromas 2.6.4 (Technelysium, South Brisbane, Australia). 


\section{qPCR analysis}

cDNA was synthesized from the extracted RNA using RevertAid first strand cDNA synthesis kit. Genomic DNA was extracted using Wizard genomic DNA purification kit (Promega, Madison, WI, USA). qPCR analysis was conducted using the CFX Connect real-time PCR detection system (Bio-Rad, Hercules, CA, USA) and SYBR Premix Ex Taq (Takara) according to the manufacturer's instructions. 18S ribosomal RNA and GAPDH were used as housekeeping controls for human and mouse cells, respectively. The sequences of the PCR primers for each TP53 exon were obtained from the International Agency for Research on Cancer TP53 database (p53.iarc.fr). The other primers used for the analyses are listed in Supplementary Table 2.

\section{Western blot analysis}

Protein extracts from whole-cell lysates were prepared using RIPA lysis buffer (LPS Solution) containing $1 \mathrm{mM} \beta$-glycerophosphate, $2.5 \mathrm{mM}$ sodium pyrophosphate, $1 \mathrm{mM} \mathrm{NaF}$, $1 \mathrm{mM} \mathrm{Na} \mathrm{VO}_{4}$, and protease inhibitor (Roche, Basel, Swiss). Protein was quantified using the Bradford assay (Bio-Rad) according to the manufacturer's protocol. Protein extracts $(30 \mu \mathrm{g})$ were separated by $12 \%$ sodium dodecyl sulfate-polyacrylamide gel electrophoresis and transferred to polyvinylidene difluoride membranes (Pall, Cortland, NY, USA). The membranes were blocked with $5 \%$ non-fat skim milk, followed by incubation with primary antibodies. After incubation with HRP-conjugated secondary antibodies, target proteins were visualized using the SuperSignal West Pico chemiluminescent substrate (Elpis Biotech, Daejeon, Korea).

\section{Flow cytometry of the immune cells}

Brain xenograft tumors were resected from mice grafted with MD13 $(n=3)$ or 157NS $(n=3)$. Similarly, brains were collected from mice without engraftment $(n=6)$, and blood and spleens were collected from all animals. Trypsinmediated digestion was used to dissociate brain and brain tumor tissues into single cells, and splenocytes were physically dissected from the spleens. Erythrocytes were eliminated from the tissue-derived cells and blood using Gey's balanced salt solution. Subsequently, the cells were incubated with the following antibodies (1:200 in RPMI medium containing 5\% fetal bovine serum): CD45-APC, TMEM119, Ly6G-PE, CD11b-FITC, and F4/80-PE. Cells were washed with PBS and additionally incubated with Alexa 594 anti-rabbit $\operatorname{IgG}$ (1:500) for detecting microglia. Flow cytometry analysis was performed using FACSVerse (BD Biosciences).

\section{Antibodies}

The following antibodies were used in this study: total p53 (DO-1; Santa Cruz Biotechnology, Dallas, TX, USA), phospho-p53 (ser15) (9284; Cell Signaling Technology, Danvers, MA, USA), p21 ${ }^{\mathrm{CIP1}}$ (C-19; Santa Cruz Biotechnology), $\beta$-actin (C4; Santa Cruz Biotechnology), CD45 (2B11 + PD7/26; Agilent, Santa Clara, CA, USA), CD45APC (561018; BD Biosciences, San Jose, CA, USA), human IBA1 (ab5076; Abcam, Cambridge, UK), mouse IBA1 (019-19741; Wako, Osaka, Japan), TMEM119 (ab210405, Abcam), Ly6G (551459; BD Biosciences), Ly6G-PE (551461; BD Biosciences), CD11b-FITC (AAS28610c; Antibody Verify, Las Vegas, NV, USA), CCL2 (ab9669; Abcam), TNF $\alpha$ (559071; BD Biosciences), HRP-conjugated goat anti-rabbit $\operatorname{IgG}(\mathrm{H}+\mathrm{L})$ (Thermo Fisher Scientific), HRP-conjugated goat anti-mouse $\mathrm{IgG}(\mathrm{H}$ + L) (Thermo Fisher Scientific), biotinylated anti-rabbit IgG (Vector Laboratories), biotinylated anti-mouse IgG (Vector Laboratories), biotinylated anti-goat IgG (Vector Laboratories), Alexa 594 anti-mouse $\operatorname{IgG}$ (Invitrogen), Alexa 594 anti-rabbit IgG (Invitrogen), and Alexa 488 antirabbit IgG (Invitrogen).

\section{Treatment with IR}

Cells were harvested and stained with trypan blue to count living cells. They were subsequently treated with $10 \mathrm{~Gy}$ of gamma irradiation by exposure to ${ }^{137} \mathrm{Cs}$ isotope using an IBL 473C irradiator (Pharmalucence, Billerica, MA, USA). After $16 \mathrm{~h}$, the cells were harvested to extract protein and RNA for western blot and qPCR, respectively.

\section{Immune cell infiltration assay}

For the BV2 microglial infiltration assay, $6 \times 10^{5} \mathrm{U} 87 \mathrm{MG}$ cells were seeded on a $60-\mathrm{mm}$ culture dish. After $24 \mathrm{~h}$, the culture medium was replaced with $2 \mathrm{~mL}$ DMEM without supplementation. The culture medium was harvested after $24 \mathrm{~h}$ and used as CM in further analysis. BV2 cells were serum-starved in DMEM without supplementation for $24 \mathrm{~h}$; then, $5 \times 10^{4}$ cells per well were loaded into the upper chamber of a $6.5-\mathrm{mm}$ Transwell with $8.0-\mu \mathrm{m}$ pore (Corning, Corning, NY, USA) coated with 10\% Matrigel (BD Biosciences) and $0.2 \%$ gelatin (Sigma-Aldrich). The lower chamber was filled with $700 \mu \mathrm{L} \mathrm{CM}$. After $24 \mathrm{~h}$, cells on the bottom of the upper chamber, which had infiltrated across the Matrigel, were stained with crystal violet (SigmaAldrich). Staining intensity was quantified using the Metamorph Offline software.

HL60 cells were differentiated into neutrophils and macrophages by treatment with $1.25 \%$ DMSO (SigmaAldrich) and $20 \mathrm{nM}$ PMA (Sigma-Aldrich) for $72 \mathrm{~h}$, 
respectively. Differentiated HL60 cells were confirmed by Diff-Quik staining (Sysmex, Kobe, Japan). Microscopic images were acquired using an Axio Imager M1 upright microscope. CM was generated as described above; however, RPMI-1640 medium was used instead of DMEM. HL60 cells $\left(1 \times 10^{5}\right)$ were loaded into the upper chamber of a $6.5-\mathrm{mm}$ Transwell with $5.0-\mu \mathrm{m}$ pore (Corning), and the lower chamber was filled with $800 \mu \mathrm{L} \mathrm{CM}$. After $24 \mathrm{~h}$, the cells that had infiltrated into the $\mathrm{CM}$ of the lower chamber were harvested and counted using a LUNA-II automated cell counter (Logos Biosystems, Anyang, Korea).

\section{In vitro cell growth analysis}

For in vitro cell growth analysis, $T P 53^{R 248 L}$-overexpressing U87MG, with or without $C C L 2$ knockdown, were seeded onto a 6 -well plate $\left(1.0 \times 10^{5}\right.$ cells per well). Cell confluence was quantified using the IncuCyte ZOOM system (ESSEN Bioscience, Ann Arbor, MI, USA).

\section{Inhibition of NFKB signaling}

To block NFkB signaling, $10 \mu \mathrm{M}$ Bay11-7085 (Merck, Darmstadt, German) was added to serum-free DMEM. Cells were harvested after $24 \mathrm{~h}$, RNA was extracted, and cDNA was sequentially synthesized and processed for qPCR as described above.

\section{Bioinformatics analysis}

RNA sequencing was performed by the Beijing Genomics Institute (BGI, Shenzen, China). Raw fragments per kilobase million (FPKM) and probability values $\left(P_{\mathrm{A}}\right)$ calculated by the performer's algorithm were used for further analyses. Genes with $P_{\mathrm{A}}>0.7$, the expression levels of which were increased by more than 2-fold after overexpression of $T P 53^{R 248 L}$, were grouped and designated as the $T P 53^{R 248 L}$ signature (GSE101980).

A gene expression dataset of patients with GBM and a combined dataset of patients with LGG and GBM, compiled by TCGA, was downloaded from the GlioVis data portal [53]. Patient gene expression datasets of breast invasive carcinoma, bladder urothelial carcinoma, colorectal adenocarcinoma, and ovarian cystadenocarcinoma were compiled by TCGA and downloaded from cBioPortal. A gene expression dataset of patient-derived GBM lines was provided by Dr. Ichiro Nakano. A dataset of sub-tumor localization-specific gene expression was downloaded from the IVY GAP.

Gene set enrichment analysis (GSEA) was conducted using GSEA version 3.0 (Broad Institute, Cambridge, MA, USA) and the RNA sequencing data consisting of triplicate FPKM values for each gene. Hallmark signatures, including
TNFA signaling via NFKB, interferon alpha and gamma responses, and inflammatory response, were obtained from Molecular Signature Database (MSigDB). The gene sets of Chemotaxis and Regulation of neutrophil chemotaxis were also downloaded from MSigDB. The result was considered statistically significant when the false discovery rate was $<0.3$. Single-sample GSEA (ssGSEA) was conducted with ssGSEAProjection version 8 (Broad Institute). To investigate the grade-dependent enrichment of the TP53 $3^{R 248 L}$ signature, as well as correlations among gene expression and enrichment of gene sets, gene expression values and enrichment scores were normalized as $z$-scores. The extent of correlation is displayed as a correlation coefficient $(r)$.

Kaplan-Meier survival plots were generated using SPSS statistics (IBM, Armonk, NY, USA). TAN-related and TAM-related genes were quoted from previous studies [32, 33], and the correlations were investigated using the expression dataset of GBM patients. Heat maps were generated using the Genesis software [54].

\section{Statistics}

Data presented as bar graphs are mean \pm standard error of the mean (SEM). Data were analyzed using two-tailed Student's $t$-tests. $P$-values $<0.05$ were considered statistically significant. The statistics for bioinformatics analyses are explained above (see "Bioinformatics analysis" in Materials and methods).

Supplementary information is available at Cell Death and Differentiation's website.

Acknowledgements We thank Dr. Joonbeom Bae, Dr. Chang-Yong Choi, and Sang-Pil Choi from Korea University for their advice on experiments related to immune cells, and Ock Ran Kim (Seoul National University Hospital) for advice on histological analyses. This work was supported by grants to Hyunggee Kim from the National Research Foundation (NRF) (2015R1A5A1009024 and 2017M3A9A8031425), Next-Generation Biogreen 21 Program (PJ01107701), Brain Korea 21 Plus, and Korea University, from the Korea Health Industry Development Institute (KHIDI) (HI14C3418) to D-HN, and from the NRF (2016M3C7A1913844) to SHK. The bio-specimens for this study were provided by Samsung Medical Center Biobank.

\section{Compliance with ethical standards}

Conflict of interest The authors declare that they have no conflict of interest.

\section{References}

1. Omuro A, DeAngelis LM. Glioblastoma and other malignant gliomas: a clinical review. J Am Med Assoc. 2013;310:1842-50.

2. Ostrom QT, Gittleman H, Liao P, Rouse C, Chen Y, Dowling J, et al. CBTRUS statistical report: primary brain and central nervous system tumors diagnosed in the United States in 2007-2011. Neuro Oncol. 2014;16:iv1-63. 
3. Brennan CW, Verhaak RG, McKenna A, Campos B, Noushmehr H, Salama SR, et al. The somatic genomic landscape of glioblastoma. Cell. 2013;155:462-77.

4. Verhaak RG, Hoadley KA, Purdom E, Wang V, Qi Y, Wilkerson $\mathrm{MD}$, et al. Integrated genomic analysis identifies clinically relevant subtypes of glioblastoma characterized by abnormalities in PDGFRA, IDH1, EGFR, and NF1. Cancer Cell. 2010;17:98-110.

5. Chinot OL, Wick W, Mason W, Henriksson R, Saran F, Nishikawa $\mathrm{R}$, et al. Bevacizumab plus radiotherapy-temozolomide for newly diagnosed glioblastoma. N Engl J Med. 2014;370:709-22.

6. Olivier M, Hollstein M, Hainaut P. TP53 mutations in human cancers: origins, consequences, and clinical use. Cold Spring Harb Perspect Biol. 2010;2:a001008.

7. Bieging KT, Mello SS, Attardi LD. Unravelling mechanisms of p53-mediated tumour suppression. Nat Rev Cancer. 2014;14:359-70.

8. The Cancer Genome Atlas Research Network. Integrated genomic analyses of ovarian carcinoma. Nature. 2011;474:609-15.

9. The Cancer Genome Atlas Research Network. Comprehensive genomic characterization of squamous cell lung cancers. Nature. 2012;489:519-25.

10. The Cancer Genome Atlas Research Network. Integrated genomic characterization of papillary thyroid carcinoma. Cell. 2014;159:676-90.

11. The Cancer Genome Atlas Research Network. Comprehensive molecular characterization of clear cell renal cell carcinoma. Nature. 2013;499:43-9.

12. Muller PA, Vousden KH. Mutant p53 in cancer: new functions and therapeutic opportunities. Cancer Cell. 2014;25:304-17.

13. Alexandrova EM, Yallowitz AR, Li D, Xu S, Schulz R, Proia DA, et al. Improving survival by exploiting tumour dependence on stabilized mutant p53 for treatment. Nature. 2015;523:352-6.

14. Soragni A, Janzen DM, Johnson LM, Lindgren AG, Thai-Quynh Nguyen A, Tiourin E, et al. A designed inhibitor of p53 aggregation rescues p53 tumor suppression in ovarian carcinomas. Cancer Cell. 2016;29:90-103.

15. Shalapour S, Karin M. Immunity, inflammation, and cancer: an eternal fight between good and evil. J Clin Invest. 2015;125:3347-55.

16. Terzic J, Grivennikov S, Karin E, Karin M. Inflammation and colon cancer. Gastroenterology. 2010;138:2101-14.

17. Oeckinghaus A, Ghosh S. The NF- $\mathrm{BB}$ family of transcription factors and its regulation. Cold Spring Harb Perspect Biol. 2009;1: a000034.

18. Granata F, Frattini A, Loffredo S, Staiano RI, Petraroli A, Ribatti $\mathrm{D}$, et al. Production of vascular endothelial growth factors from human lung macrophages induced by group IIA and group $\mathrm{X}$ secreted phospholipases A2. J Immunol. 2010;184:5232-41.

19. Tjiu JW, Chen JS, Shun CT, Lin SJ, Liao YH, Chu CY, et al. Tumor-associated macrophage-induced invasion and angiogenesis of human basal cell carcinoma cells by cyclooxygenase-2 induction. J Investig Dermatol. 2009;129:1016-25.

20. Pyonteck SM, Akkari L, Schuhmacher AJ, Bowman RL, Sevenich L, Quail DF, et al. CSF-1R inhibition alters macrophage polarization and blocks glioma progression. Nat Med. 2013;19:1264-72.

21. Zhu Y, Knolhoff BL, Meyer MA, Nywening TM, West BL, Luo $\mathrm{J}$, et al. CSF1/CSF1R blockade reprograms tumor-infiltrating macrophages and improves response to $\mathrm{T}$ cell checkpoint immunotherapy in pancreatic cancer models. Cancer Res. 2014;74:5057-69.

22. Whibley C, Pharoah PD, Hollstein M. p53 polymorphisms: cancer implications. Nat Rev Cancer. 2009;9:95-107.

23. Abbas T, Sivaprasad U, Terai K, Amador V, Pagano M, Dutta A. PCNA-dependent regulation of p21 ubiquitylation and degradation via the CRL4Cdt2 ubiquitin ligase complex. Genes Dev. 2008;22:2496-506.
24. Bendjennat M, Boulaire J, Jascur T, Brickner H, Barbier V, Sarasin $\mathrm{A}$, et al. UV irradiation triggers ubiquitin-dependent degradation ofp21(WAF1) to promote DNA repair. Cell. 2003;114:599-610.

25. Stuart SA, Wang JY. Ionizing radiation induces ATMindependent degradation of p21Cip1 in transformed cells. J Biol Chem. 2009;284:15061-70.

26. Bennett ML, Bennett FC, Liddelow SA, Ajami B, Zamanian JL, Fernhoff NB, et al. New tools for studying microglia in the mouse and human CNS. Proc Natl Acad Sci USA. 2016;113: E1738-46.

27. Tarella C, Ferrero D, Gallo E, Pagliardi GL, Ruscetti FW. Induction of differentiation of HL-60 cells by dimethyl sulfoxide: evidence for a stochastic model not linked to the cell division cycle. Cancer Res. 1982;42:445-9.

28. Padilla PI, Wada A, Yahiro K, Kimura M, Niidome T, Aoyagi H, et al. Morphologic differentiation of HL-60 cells is associated with appearance of RPTPbeta and induction of Helicobacter pylori VacA sensitivity. J Biol Chem. 2000;275:15200-6.

29. Bossen C, Ingold K, Tardivel A, Bodmer JL, Gaide O, Hertig S, et al. Interactions of tumor necrosis factor (TNF) and TNF receptor family members in the mouse and human. J Biol Chem. 2006;281:13964-71.

30. Tang X, Asano M, O'Reilly A, Farquhar A, Yang Y, Amar S. p53 is an important regulator of CCL2 gene expression. Curr Mol Med. 2012;12:929-43.

31. Cooks T, Pateras IS, Tarcic O, Solomon H, Schetter AJ, Wilder S, et al. Mutant p53 prolongs NF-kappaB activation and promotes chronic inflammation and inflammation-associated colorectal cancer. Cancer Cell. 2013;23:634-46.

32. Eruslanov EB, Bhojnagarwala PS, Quatromoni JG, Stephen TL, Ranganathan A, Deshpande C, et al. Tumor-associated neutrophils stimulate $\mathrm{T}$ cell responses in early-stage human lung cancer. J Clin Invest. 2014;124:5466-80.

33. Hartwig T, Montinaro A, von Karstedt S, Sevko A, Surinova S, Chakravarthy A, et al. The TRAIL-induced cancer secretome promotes a tumor-supportive immune microenvironment via CCR2. Mol Cell. 2017;65:730-42.e735.

34. Wang X, Chen JX, Liu JP, You C, Liu YH, Mao Q. Gain of function of mutant TP53 in glioblastoma: prognosis and response to temozolomide. Ann Surg Oncol. 2014;21:1337-44.

35. Shiraishi S, Tada K, Nakamura H, Makino K, Kochi M, Saya H, et al. Influence of p53 mutations on prognosis of patients with glioblastoma. Cancer. 2002;95:249-57.

36. Pollack IF, Finkelstein SD, Woods J, Burnham J, Holmes EJ, Hamilton RL, et al. Expression of p53 and prognosis in children with malignant gliomas. N Engl J Med. 2002;346:420-7.

37. Lee SY. Temozolomide resistance in glioblastoma multiforme. Genes Dis. 2016;3:198-210.

38. Kyritsis AP, Bondy ML, Hess KR, Cunningham JE, Zhu D, Amos $\mathrm{CJ}$, et al. Prognostic significance of p53 immunoreactivity in patients with glioma. Clin Cancer Res. 1995;1:1617-22.

39. Jin Y, Xiao W, Song T, Feng G, Dai Z. Expression and prognostic significance of p53 in glioma patients: a meta-analysis. Neurochem Res. 2016;41:1723-31.

40. Arimappamagan A, Somasundaram K, Thennarasu K, Peddagangannagari S, Srinivasan $\mathrm{H}$, Shailaja BC, et al. A fourteen gene GBM prognostic signature identifies association of immune response pathway and mesenchymal subtype with high risk group. PLoS ONE. 2013;8:e62042.

41. Mostafa H, Pala A, Högel J, Hlavac M, Dietrich E, Westhoff MA, et al. Immune phenotypes predict survival in patients with glioblastoma multiforme. J Hematol Oncol. 2016;9:77.

42. Wang PF, Song HW, Cai HQ, Kong LW, Yao K, Jiang T, et al. Preoperative inflammation markers and IDH mutation status predict glioblastoma patient survival. Oncotarget. 2017;8:50117-23. 
43. Wang Q, Hu B, Hu X, Kim H, Squatrito M, Scarpace L, et al. Tumor evolution of glioma-intrinsic gene expression subtypes associates with immunological changes in the microenvironment. Cancer Cell. 2017;32:42-56. e46.

44. van Horssen R, ten Hagen TL, Eggermont AM. TNF-alpha in cancer treatment: molecular insights, antitumor effects, and clinical utility. Oncologist. 2006;11:397-408.

45. Carro MS, Lim WK, Alvarez MJ, Bollo RJ, Zhao X, Snyder EY, et al. The transcriptional network for mesenchymal transformation of brain tumours. Nature. 2010;463:318-25.

46. Bhat Krishna PL, Balasubramaniyan V, Vaillant B, Ezhilarasan R, Hummelink K, Hollingsworth F, et al. Mesenchymal differentiation mediated by NF- $\mathrm{KB}$ promotes radiation resistance in glioblastoma. Cancer Cell. 2013;24:331-46.

47. Kim SH, Ezhilarasan R, Phillips E, Gallego-Perez D, Sparks A, Taylor D, et al. Serine/threonine kinase MLK4 determines mesenchymal identity in glioma stem cells in an NF-kBdependent manner. Cancer Cell. 2016;29:201-13.

48. Han J, Chu J, Keung Chan W, Zhang J, Wang Y, Cohen JB, et al. CAR-engineered NK cells targeting wild-type EGFR and EGFRvIII enhance killing of glioblastoma and patient-derived glioblastoma stem cells. Sci Rep. 2015;5:11483.
49. Zhang J, Sarkar S, Cua R, Zhou Y, Hader W, Yong VW. A dialog between glioma and microglia that promotes tumor invasiveness through the CCL2/CCR2/interleukin-6 axis. Carcinogenesis. 2012;33:312-9.

50. Roca H, Varsos ZS, Sud S, Craig MJ, Ying C, Pienta KJ. CCL2 and interleukin- 6 promote survival of human $\mathrm{CD} 11 \mathrm{~b}+$ peripheral blood mononuclear cells and induce M2-type macrophage polarization. J Biol Chem. 2009;284:34342-54.

51. Chang AL, Miska J, Wainwright DA, Dey M, Rivetta CV, Yu D, et al. CCL2 produced by the glioma microenvironment is essential for the recruitment of regulatory $\mathrm{T}$ cells and myeloid-derived suppressor cells. Cancer Res. 2016;76:5671.

52. Bachoo RM, Maher EA, Ligon KL, Sharpless NE, Chan SS, You $\mathrm{MJ}$, et al. Epidermal growth factor receptor and Ink4a/Arf: convergent mechanisms governing terminal differentiation and transformation along the neural stem cell to astrocyte axis. Cancer Cell. 2002;1:269-77.

53. Bowman RL, Wang Q, Carro A, Verhaak RGW, Squatrito M. GlioVis data portal for visualization and analysis of brain tumor expression datasets. Neuro Oncol. 2017;19:139-41.

54. Sturn A, Quakenbush J, Trajanoski Z. Genesis: cluster analysis of microarray data. Bioinformatics. 2002;18:207-8. 